\title{
Blow-up results for vector-valued nonlinear heat equations with no gradient structure
}

by

\author{
Hatem ZAAG \\ Université de Cergy-Pontoise, Ecole Normale Supérieure, \\ 45, rue d'Ulm, 75005 Paris. \\ E-mail:zaag@dmi.ens.fr
}

ABSTRACT. - We construct a blow-up solution for the following nonlinear complex equation:

$$
u_{t}=\Delta u+(1+i \delta)|u|^{p-1} u, \quad u \in \mathbb{C} .
$$

We also find the asymptotic profile near the singularity, and generalize the result to other vector-valued equations. (C) Elsevier, Paris

Key words: 35K: parabolic systems, 35B40: asymptotic behavior of solutions.

RÉSUMÉ. - On construit une solution explosive de l'équation non linéaire complexe suivante :

$$
u_{t}=\Delta u+(1+i \delta)|u|^{p-1} u, \quad u \in \mathbb{C} .
$$

On donne aussi son profil asymptotique au voisinage de la singularité, et on généralise le résultat à d'autres équations à valeurs vectorielles. (C) Elsevier, Paris

\section{INTRODUCTION}

We are interested in the following reaction-diffusion equation:

$$
\frac{\partial u}{\partial t}=\Delta u+(1+i \delta)|u|^{p-1} u, u(0, x)=u_{0}(x),
$$

\footnotetext{
* AMS Classification: $35 \mathrm{~K}$ : parabolic systems, 35 B 40: asymptotic behavior of solutions.
} 
where, $\delta \in \mathbb{R}, p \in(1,+\infty), p<(N+2) /(N-2)$ if $N \geq 3$, and $u_{0} \in H=W^{1, p+1}\left(\mathbb{R}^{N}, \mathbb{C}\right) \cap L^{\infty}\left(\mathbb{R}^{N}, \mathbb{C}\right)$.

(1) is a special case of the vector-valued equation:

$$
\frac{\partial u}{\partial t}=\Delta u+F(u), u(x, 0)=u_{0}(x),
$$

where $u(t): x \in \mathbb{R}^{N} \rightarrow \mathbb{R}^{M}, F: \mathbb{R}^{M} \rightarrow \mathbb{R}^{M}$ is regular and $F$ is not necessarily a gradient.

For simplicity, we focus on the study of (1) (results for equation (2) will also be presented in section 5).

Equation (1) appears in the study of various physical problems (plasma physics, nonlinear optics). See for example Levermore and Oliver [15] and the references inside. Blow-up results for vector-valued equations have been intensively studied in differential geometry. See for example a review paper by Hamilton [12].

The Cauchy problem for equation (1) can be solved in $H . u(t)$, solution of (1) would exist either on $[0,+\infty)$ (global existence), or only on $[0, T)$, with $0<T<+\infty$. In this case, $|u(t)|_{H} \rightarrow+\infty$ when $t \rightarrow T$, we say: $u(t)$ blows-up in finite time $T$ in $H$. In this paper, we are interested in the finite time blow-up for equation (1).

If $\delta=0$ and $u_{0}(x) \in \mathbb{R}$, then (1) can be considered as real-valued. Blow-up in this real case has been studied by various authors. Relying on the use of monotony properties and maximum principle, Ball [1] and Levine [16] find in this case obstructions to the global in time existence for (1). Other authors investigated the asymptotic behavior at blow-up of blow-up solutions of (1), $\delta=0$. See for example Weissler [20], see for a study in the scale of similarity variables Giga and Kohn [11], [10], [9], Filippas and Kohn [5], Filippas and Merle [6],... The notion of asymptotic profile (that is a function from which, after a time dependent scaling, $u(t)$ approaches as $t \rightarrow T$ ) appears also in various papers: see for example Bricmont and Kupiainen [4], [3], Berger and Kohn [2] for a numerical study. In the scalar case and in one dimension, Herrero and Velazquez give a classification of possible blow-up profiles. They use the maximum principle and the decay in time of the number of oscillations of the solution. Some of their results are generalized to $N$ dimensions in [19].

Most of the techniques used for $\delta=0$ in the cited papers can not be applied in the case $\delta \neq 0$, since (1) is complex-valued (no maximum principle applied), and the equation does not derive from a gradient.

Another method has been introduced in [18] in the case $\delta=0$ (see also [4]): Once an asymptotic profile is derived formally for (1), the existence 
of a solution $u(t)$ which blows-up in finite time with the suggested profile is proved rigorously, using a nonlinear analysis of equation (2) near the given profile. This approach which does not use maximum principle allows us to find blow-up solutions for vector-valued heat equations (even with no gradient structure). In this paper, we aim at adapting this method to show the existence of a blow-up solution for equation (1) with $\delta \neq 0$.

Let us remark that the scalar case provides us with a blow-up solution if $\delta=0$. Unfortunately, this result is a one dimensional result and it fails when we perturb slightly the nonlinearity. Indeed, let us mention the case of the following vectorial equation:

$$
\frac{\partial u}{\partial t}=\Delta u+|u|^{p-1} u+i|u|^{q-1} u, u_{\mid \partial \Omega}=0
$$

with $1<q<(p+1) / 2$, the method of Ball [1] yields a blow-up solution $u(t): \Omega \rightarrow \mathbb{C}$ where $\Omega$ is a bounded domain of $\mathbb{R}^{N}$, see appendix $\mathrm{A}$ for details.

We show that there exists $\delta_{0}>0$ such that for each $\delta \in\left[-\delta_{0}, \delta_{0}\right]$, equation (1) has a blow-up solution. We give in addition a precise description of its blow-up behavior. Indeed,

THEOREM 1 (Existence of a blow-up solution for equation (1) for small $\delta)$. - There exists $\delta_{0}>0$ such that for each $\delta \in\left[-\delta_{0}, \delta_{0}\right]$, there exist initial data $u_{0}$ such that equation (1) has a blow-up solution.

This Theorem follows directly from the following proposition which specifies the behavior of $u(t)$ near blow-up. Indeed, up to a time dependent scaling, $u(t)$ approaches a universal profile

$$
\left(p-1+\frac{(p-1)^{2}}{4\left(p-\delta^{2}\right)}|z|^{2}\right)^{-\frac{1+i \delta}{p-1}}
$$

when $t \rightarrow T$. More precisely:

Proposition 1 (Existence of a blow-up solution for equation (1) with the profile (4)). - There exist $\delta_{0}>0, T_{0}>0$ such that for each $\delta \in\left[-\delta_{0}, \delta_{0}\right]$, for each $T \in\left(0, T_{0}\right]$, for each $a \in \mathbb{R}^{N}$,

i) there exist initial data $u_{0}$ such that equation (1) has a blow-up solution $u(x, t)$ on $\mathbb{R}^{N} \times[0, T)$ which blows-up in finite time $T$ at only one blow-up point: $a$.

ii) moreover, we have

$$
\lim _{t \rightarrow T}\left\|(T-t)^{\frac{1+i \delta}{p+1}} u\left(a+((T-t)|\log (T-t)|)^{\frac{1}{2}} z, t\right)-f_{\delta}(z)^{1+i \delta}\right\|_{L^{\infty}\left(\mathbb{R}^{N}\right)}-0
$$


with

$$
f_{\delta}(z)=\left(p-1+\frac{(p-1)^{2}}{4\left(p-\delta^{2}\right)}|z|^{2}\right)^{-\frac{1}{p-1}} .
$$

iii) There exists $u_{*} \in \mathcal{C}\left(\mathbb{R}^{N} \backslash\{a\}, \mathbb{C}\right)$ such that $u(x, l) \rightarrow u_{*}(x)$ as $l \rightarrow T$ uniformly on compact subsets of $\mathbb{R}^{N} \backslash\{a\}$, and

$$
u_{*}(x) \sim\left[\frac{8\left(p-\delta^{2}\right)|\log | x-a||}{(p-1)^{2}|x-a|^{2}}\right]^{\frac{1+i \delta}{p-1}} \text { as } x \rightarrow a
$$

Remark. - Estimate (5) is really uniform in $z \in \mathbb{R}^{N}$. In previous papers dealing with the case $\delta=0$, only Bricmont and Kupiainen [4] and Merle and Zaag [18] give such a uniform convergence. In most papers, the same kind convergence is proved, but only uniformly on smaller subsets (for $|z| \leq C / \sqrt{|\log (T-t)|}$ in $[5], \ldots)$.

Remark. - In fact, we show that property iii) is a consequence of ii). We want to point out that for the heat equation $(\delta=0)$, iii) was known just in dimension one using the decay in time of the number of oscillations of the solution (Cf Herrero and Velazquez [13]).

Remark. - To prove Proposition 1, we linearize in a way equation (1) around $f_{\delta}^{1+i \delta}$, and give a nonlinear finite dimensional reduction of the problem. Then, we solve the finite dimensional problem using index theory. The proof is more difficult than in [18], because of the vectorial structure, the presence of a coupling between coordinates, and the presence of one more neutral direction. These techniques give then as in [18] a stability result with respect to the initial data of the behavior described in Proposition 1 (see section 5).

Remark. - Center manifold theory do not apply here. It fails to give a uniform estimate such as ii). One can point out that even if it works, a center manifold theory gives a convergence only uniform in the region $\{|z| \sqrt{|\log (T-t)|} \leq C\}$. For discussion in the case $\delta=0$, see Filippas and Kohn [5], page 834-835.

Remark. - We see from (6) that $0<\delta_{0}<\sqrt{p}$. Since equation (1) is rotation invariant, for each $\omega \in S^{1}$, we can find initial data $u_{0}$ such that the corresponding solution has the profile $f_{\delta}^{1+i \delta} \omega$.

From this result, one can ask: what happens for $\delta>\delta_{0}$ ? Does equation (1) still have blow-up solutions? We conjecture the existence of $\hat{\delta}_{0}>0$ such that for $|\delta|<\hat{\delta}_{0}$, equation (1) has blow-up solutions, while for $|\delta|>\delta_{0}$, no blow-up is possible for solutions of equation (1). That is, all solutions 
are globally defined. Indeed, from the formal asymptotic analysis, one can remark that for $|\delta|>\sqrt{p}, \int_{\delta}^{1+i \delta}$ is no longer bounded, and the analysis fails. Another question arises: what happens with the critical value $\delta=\hat{\delta}_{0}$ ? Unfortunately, we are not able here to give a precise value of $\hat{\delta}_{0}$ and a rigorous proof of what is conjectured.

As an extension of Theorem 1, one can mention that using the same techniques, we have the same result for the following vector-valued equation:

$$
\frac{d u}{d t}=\Delta u+|u|^{p-1} u+G(u), u(x, 0)=u_{0}(x)
$$

where

1) $u(t): x \in \mathbb{R}^{N} \rightarrow \mathbb{R}^{M}, p \in(1,+\infty), p<(N+2) /(N-2)$ if $N \geq 3$, $u_{0} \in H=W^{1, p+1}\left(\mathbb{R}^{N}, \mathbb{R}^{M}\right) \cap L^{\infty}\left(\mathbb{R}^{N}, \mathbb{R}^{M}\right)$,

2) $G: \mathbb{R}^{M} \rightarrow \mathbb{R}^{M}$ is a perturbation of $|u|^{p-1} u$ satisfying: $G(u)=$ $G_{1}\left(|u|^{2}\right) u,|G(u)| \leq C|u|^{r},\left|G\left(\lambda u_{1}\right)-G\left(\lambda u_{2}\right)\right| \leq C \lambda^{r}\left|u_{1}-u_{2}\right|$ for $\left|u_{1}\right|,\left|u_{2}\right| \leq 1, \lambda \geq 1, r \in[1, p), G_{1}: \mathbb{R}^{+} \rightarrow \mathbb{R}^{+}$,

Indeed,

THEOREM 2 (Existence of a blow-up solution for equation (8)). - There exist initial data $u_{0}$ such that equation (8) has a blow-up solution.

Let us mention briefly the organization of the paper. The proof of Proposition 1 relies strongly on a double-scale description of $u(t)$, solution of (1). We first give in section 2 an equivalent formulation of the problem in the scale of the well known similarity variables (see Giga and Kohn [11],...). Then, working in the original scale, we prove in section 3 the existence of a single-point blow-up solution for equation (1) such that (5) holds. In section 4 , we return to the original scale $u(x, t)$ and use the invariance of equation (1) under the transformation $\left(t_{0}, \lambda\right) \rightarrow u_{\lambda}(x, t)=\lambda^{\frac{1+i \delta}{p-1}} u\left(\sqrt{\lambda} x, t_{0}+\lambda t\right)$ to show that estimate (5) yields the equivalent (7) for the profile $u_{*}$ in the original scale. We conclude in section 5 by giving some comments about the stability of the result of Proposition 1 and detailing the case of equation (8) $(M \geq 3)$.

Without loss of generality, we can now assume that $a=0$ and $N=1$. The same proof holds in higher dimensions (see [18] for the analysis of the case $N \geq 2$ ). We write each complex quantity (number or function) $z$ as $z=z_{1}+i z_{2}$ with $z_{1}, z_{2} \in \mathbb{R}$.

The author wants to thank Professor F. Merle for his helpful suggestions and remarks. 


\section{FORMULATION OF THE PROBLEM}

As we mentioned just before, the proof of Proposition 1 will be completed in two steps. In the first step (section 3), it is enough to construct $u(t)$ a solution of equation (1) satisfying (5), since this implies directly that $u(t)$ blows-up in finite time $T$ at only one blow-up point: 0 (parts i) and ii) of Proposition 1). Indeed, it easily follows from (5) that $\lim _{t \rightarrow T}|u(0, t)|=+\infty$, which means that $u(t)$ blows-up in time $T$ at the point 0 , and $\lim _{t \rightarrow T}(T-t)^{\frac{1}{p-1}}|u(b, t)|=0$ for $b \neq 0$, which implies in turn that $u(t)$ does not blow-up at $b \neq 0$, and therefore blows-up only at the point 0 . This last result follows directly from a Theorem by Giga and Kohn (Theorem 2.1 in [11]).

In a second step (section 4), we show how the behavior of the limiting profile $u_{*}(x)$ near the blow-up point (part iii) of Proposition 1) can be derived from the behavior of $u(t)$ as $t \rightarrow T$ given by (5).

Hence, our first goal is to construct $u(t)$ a solution of (1) satisfying (5).

To have an idea about the blow-up growth of $u$, solution of equation (1), we compare this solution with a blow-up solution of the corresponding differential equation

$$
\frac{d u}{d t}=(1+i \delta)|u|^{p-1} u
$$

This solution is $u(t)=e^{i \theta}((p-1)(T-t))^{-\frac{1+i t}{p-1}}$, with $T>0, \theta \in \mathbb{R}$.

Now, we consider $u$, a solution of equation (1) which blows-up in finite time $T>0$ at one blow-up point $0 \in \mathbb{R}$. We expect $u$ to grow with a similar rate near blow-up. If we introduce convenient "similarity variables"

$$
\begin{aligned}
y & =\frac{x}{\sqrt{T-t}} \\
s & =-\log (T-t) \\
w(y, s) & =(T-t)^{\frac{1+i \delta}{p-1}} u(x, t),
\end{aligned}
$$

then, we can look for bounded non zero solutions of the following equation (which follows from (1) through (9)):

$$
\frac{\partial w}{\partial s}=\Delta w-\frac{1}{2} y \nabla w-(1+i \delta) \frac{w}{p-1}+(1+i \delta)|w|^{p-1} w
$$

\subsection{Formal asymptotic analysis}

Since equation $(10)$ is of heat type, one can ask whether it has self-similar solutions, or at least, approximate ones. We have the following lemma: 
LEMMA 2.1 (Formal asymptotic behavior of $w$ ).

i) The only self-similar solutions $w(y, s)=v_{0}\left(\frac{y}{\sqrt{s}}\right)$ of $(10)$ are the constant ones: $v_{0} \equiv 0$, or $v_{0} \equiv \kappa e^{i \theta}$, with $\kappa=(p-1)^{-\frac{1}{p-1}}$ and $\theta \in \mathbb{R}$.

ii) If equation (10) has a solution of the form

$$
w(y, s)=\sum_{j=0}^{+\infty} \frac{1}{s^{j}} v_{j}\left(\frac{y}{\sqrt{s}}\right) .
$$

with $v_{j}$ regular and bounded, then, there exists $\theta \in \mathbb{R}$ such that

$$
v_{0}(z)=e^{i \theta}\left(p-1+\frac{(p-1)^{2}}{4\left(p-\delta^{2}\right)} z^{2}\right)^{-\frac{1+i \delta}{p-1}}=e^{i \theta} f_{\delta}(z)^{1+i \delta}
$$

where $f_{\delta}(z)^{1+i \varepsilon}$ is the suggested profile in (4).

Proof.

i) The equations satisfied by such a $v_{0}$ are

$$
0=-\frac{1}{2} z v_{0}^{\prime}(z)-(1+i \delta) \frac{v_{0}}{p-1}+(1+i \delta)\left|v_{0}\right|^{p-1} v_{0}
$$

and $-\frac{1}{2} z v_{0}^{\prime}(z)=v_{0}^{\prime \prime}(z)$. It is easy to see that the only solutions are the constant ones, and that $-\frac{v_{0}}{p-1}+\left|v_{0}\right|^{p-1} v_{0}=0$. This yields the conclusion.

ii) If we substitute the form (11) in equation (10) and set $z=\frac{y}{\sqrt{s}}$, we find (if $s \rightarrow+\infty$ ) that $v_{0}$ satisfies (13). Searching a non constant solution $v_{0}(z)=\rho(z) e^{i \theta(z)}$, with $\rho>0$, one finds that $v_{0}(z)=e^{i \theta}\left(p-1+b z^{2}\right)^{-\frac{1+i \delta}{p-1}}$, with $b>0, \theta \in \mathbb{R}$.

In fact, there is only one possible value of $b$. Indeed, if we substitute the expanded form (11) in equation (10) and compare elements of order $\frac{1}{s}$, we obtain $F(z)=0$, where $F(z)=\frac{1}{2} z v_{0}^{\prime}+v_{0}^{\prime \prime}-\frac{1}{2} z v_{1}^{\prime}-(1+i \delta) \frac{v_{1}}{p-1}+$ $(1+i \delta)\left\{(p-1)\left|v_{0}\right|^{p-3} v_{0}\left(v_{0,1} v_{1,1}+v_{0,2} v_{1,2}\right)+\left|v_{0}\right|^{p-1} v_{1}\right\}$, and $v_{j}=v_{j, 1}+$ $i v_{j, 2}, j=1,2$. According to regularization properties of equation (10), it is natural to require that $v_{1}$ is $\mathcal{C}^{3}$, which implies that $F$ is $\mathcal{C}^{2} . F^{\prime \prime}(0)=0$ implies $b=\frac{(p-1)^{2}}{4\left(p-\delta^{2}\right)}$.

Remark. - Looking for approximate solutions of (10) or for solutions of (10) in the expanded form (11) is a well known approach used in various problems such as nonlinear optics, and also nonlinear heat equations (see for instance Galaktionov, Kurdyumov and Samarskii [7] for approximate self-similar solutions in the case of global existence (in time), see also Galaktionov and Vazquez [8] where an approximate solution is shown Vol. 15. $n^{\circ} 5-1998$. 
to be an admissible blow-up profile in the case of a heat equation with $(1+u) \log ^{2}(1+u)$ as a nonlinearity). Unfortunately, computation can not be carried out easily for the form (11) in the present case, and we are unable to show the existence of a solution for equation (10) with such a form. In fact, instead of using this linear approach, we use a nonlinear one in section 3 to show that $(10)$ actually has a solution $w(y, s)$ which approaches (in $L_{y}^{\infty}$ ) $f_{\delta}\left(\frac{y}{\sqrt{s}}\right)^{1+i \delta}$ as $s \rightarrow+\infty$. This approach (instead of the linear one) yields the stability of such a solution (see section 5).

\subsection{Transformation of the problem}

Using similarity variables (see (9)), we see that proving (5) is equivalent to proving that (10) has a solution satisfying

$$
\lim _{s \rightarrow \infty}\left\|w(y, s)-f_{\delta}\left(\frac{y}{\sqrt{s}}\right)^{1+i \delta}\right\|_{L^{x}}=0
$$

where $f_{\delta}^{1+i \delta}$ is given by (4).

In order to prove this, we will not linearize equation $(10)$ around $f_{\delta}^{1+i \delta}$ as it suggested by (14), because the linear operator of the linearized equation has two neutral modes which are difficult to control. We will instead use modulation theory and take advantage of the invariance of (10) under the action of $S^{1}\left(T_{\theta_{0}}: w \rightarrow e^{i \theta_{0}} w\right.$, for each $\left.\theta_{0} \in \mathbb{R}\right)$ : in fact, we introduce $q(y, s):[-\log T,+\infty) \rightarrow \mathbb{C}$ and $\theta(s):[-\log T,+\infty) \rightarrow \mathbb{R}$ such that

$$
\left.\begin{array}{rl}
w(y, s) & =(\varphi(y, s)+q(y, s)) e^{i \theta(s)} \\
0 & =\int \chi(y, s)\left(q_{2}(y, s)-\delta q_{1}(y, s)\right) d \mu
\end{array}\right\}
$$

where

$$
\begin{gathered}
\varphi(y, s)=\kappa^{-i \delta}\left(f_{\delta}\left(\frac{y}{\sqrt{s}}\right)+\frac{\kappa}{2\left(p-\delta^{2}\right) s}\right)^{1+i \delta}, \kappa=(p-1)^{-\frac{1}{p-1}} \\
\chi(y, s)=\chi_{0}\left(\frac{|y|}{K_{0} s^{\frac{l}{2}}}\right),
\end{gathered}
$$

$\chi_{0} \in C_{0}^{\infty}([0,+\infty),[0,1])$, with $\chi_{0} \equiv 1$ on $[0,1]$ and $\chi_{0} \equiv 0$ on $[2,+\infty]$, $K_{0}$ is a constant large enough, and

$$
d \mu(y)=\frac{e^{-y^{2} / 4}}{\sqrt{4 \pi}} .
$$

The introduced liberty degree $\theta(s)$ is fixed by the second equation of (15). It will appear in the course of the proof that this second equation 
makes one of the neutral modes of the perturbation $q$ to be zero, which simplifies greatly the control of $q$.

One can remark that we don't linearize (10) around $e^{i \theta(s)} f_{\delta}^{1+i \delta}$, but around $e^{i \theta(s)} \varphi$. Up to the natural action of $S^{1}$ (multiplication by $\kappa^{-i \delta}$ ) which simplifies the study of the linear operator of the equation on $q$, these two expressions differ from each other by a term of order $\frac{1}{s}$, so that (at least) some components of $q$ are smaller that $\frac{1}{s}$, which helps to have $q(s) \rightarrow 0$ in $L_{y}^{\infty}$ as $s \rightarrow+\infty$.

Now, we claim that proving parts i) and ii) of Proposition 1 reduces to proving the following proposition:

Proposition 2.1 (Equivalent formulation of Proposition 1, i) and ii)). - There exist $\delta_{0}>0, S_{0}>0$, such that $\forall \delta \in\left[-\delta_{0}, \delta_{0}\right], \forall s_{0} \geq S_{0}$. $\exists q_{s_{0}} \in-\varphi\left(., s_{0}\right)+H$ such that the system

$$
\left.\begin{array}{rl}
\frac{\partial q}{\partial s}(y, s) & =\left\{\mathcal{L}_{\varphi}-i \frac{d \theta}{d s}\right\}(q)(y, s)+B(q)(y, s)+R(\theta, y, s) \\
0 & =\int \chi(y, s)\left(q_{2}(y, s)-\delta q_{1}(y, s)\right) d \mu(y)
\end{array}\right\}
$$

where

$$
\left.\begin{array}{rl}
\mathcal{L}_{\varphi}(q) & =\Delta q-\frac{1}{2} y \cdot \nabla q-(1+i \delta) \frac{q}{p-1} \\
& +(1+i \delta)\left\{(p-1)|\varphi|^{p-3} \varphi\left(\varphi_{1} q_{1}+\varphi_{2} q_{2}\right)+|\varphi|^{p-1} q\right\}, \\
B(q) & =(1+i \delta)\left\{|\varphi+q|^{p-1}(\varphi+q)-|\varphi|^{p-1} \varphi\right. \\
& \left.-(p-1)|\varphi|^{p-3} \varphi\left(\varphi_{1} q_{1}+\varphi_{2} q_{2}\right)-|\varphi|^{p-1} q\right\}, \\
R(\theta, y, s) & =R^{*}(y, s)-i \frac{d \theta}{d s} \varphi, \\
R^{*}(y, s) & =-\frac{\partial \varphi}{\partial s}+\Delta \varphi-\frac{1}{2} y \cdot \nabla \varphi-(1+i \delta) \frac{\varphi}{p-1}+(1+i \delta)|\varphi|^{p-1} \varphi,
\end{array}\right\}
$$

with initial data $\left(q\left(y, s_{0}\right), \theta\left(s_{0}\right)\right)=\left(q_{s_{0}}(y), 0\right)$ at $s=s_{0}$, has a unique solution $(q, \theta)$ for $s \geq s_{0}$, satisfying $\lim _{s \rightarrow+\infty}\|q(s)\|_{L^{\infty}}=0$, and $\exists \theta_{\infty} \in \mathbb{R}$ such that $\theta(s) \rightarrow \theta_{\infty}$ as $s \rightarrow+\infty$.

Indeed, due to (15), the first equation in system (19) is equivalent to (10), hence, it is equivalent to (1) (use (9)). In addition, once proposition 2.1 is proved, we have:

$$
\begin{aligned}
& \left\|w(y, s)-e^{i\left(\theta_{\infty}-\delta \log \kappa\right)} f_{\delta}\left(\frac{y}{\sqrt{s}}\right)^{1+i \delta}\right\|_{L^{\infty}} \\
& \leq\left\|e^{i \theta(s)}(q(y, s)+\varphi(y, s))-e^{i\left(\theta_{\infty}-\delta \log \kappa\right)} f_{\delta}\left(\frac{y}{\sqrt{s}}\right)^{1+i \delta}\right\|_{L^{\infty}} \text { (use (15)) }
\end{aligned}
$$

Vol. 15, n० $5-1998$. 


$$
\begin{aligned}
\leq & \|q(s)\|_{L^{\infty}}+\left\|\left(e^{i \theta(s)}-e^{i \theta x}\right) \varphi(y, s)\right\|_{L^{x}} \\
& +\left\|e^{i \theta \infty}\left(\varphi(y, s)-\kappa^{-i \delta} f_{\delta}\left(\frac{y}{\sqrt{s}}\right)^{1+i \delta}\right)\right\|_{L^{x}} \\
\leq & \|q(s)\|_{L^{\infty}}+C\left|\theta(s)-\theta_{\infty}\right|+C s^{-1} \rightarrow 0 \text { as } s \rightarrow+\infty \text { (see (16)). }
\end{aligned}
$$

Therefore, $w(y, s)$ approaches $e^{i\left(\theta_{x}-\delta \log \kappa\right)} \int_{\delta}\left(\frac{y}{\sqrt{s}}\right)^{1+i \delta}$ in $L^{\infty}(\mathbb{R})$ as $s \rightarrow+\infty$. Since $(10)$ is rotation invariant, we can replace $w$ by $e^{-i\left(\theta_{x}-\delta \log \kappa\right)} w$ to obtain (14), which is equivalent to (5) through similarity variables (see (9)).

Hence, we must study system (19) for $(q, \theta) \in L^{\infty}(\mathbb{R}) \times \mathbb{R}$ to solve the problem. Its evolution is mostly influenced by its linear part $\mathcal{L}_{\varphi, \theta}(q)=\left(\mathcal{L}_{\varphi}-i \frac{d \theta}{d s}\right)(q)$. Let us study more carefully this operator. $\mathcal{L}_{\varphi, \theta}$ is a $\mathbb{R}$-linear operator defined on $\mathcal{D}\left(\mathcal{L}_{\varphi, \theta}\right) \subset L^{2}(\mathbb{R}, \mathbb{C}, d \mu)$. Since we are interested in the behavior of $(q(s), \theta(s))$ in $L^{\infty}(\mathbb{R}) \times \mathbb{R}$ as $s \rightarrow+\infty$, let us consider the limit as $s \rightarrow+\infty$ of $\mathcal{L}_{\varphi, \theta}(r)$ for a fixed $r \in L^{\infty}(\mathbb{R}, \mathbb{C})$ (note that $L^{\infty}(\mathbb{R}, \mathbb{C}) \subset L^{2}(\mathbb{R}, \mathbb{C}, d \mu)$ ).

Since $\theta(s)$ will be shown to have a limit when $s \rightarrow+\infty$, we can think that the effect of $\frac{d \theta}{d s}$ appearing in the expression of $\mathcal{L}_{\varphi, \theta}$ (see (20)) will be negligible. Therefore, $\mathcal{L}_{\varphi, \theta}(r) \rightarrow \tilde{\mathcal{L}}(r)=\Delta r-\frac{1}{2} y \cdot \nabla r+(1+i \delta) r_{1}$ as $s \rightarrow+\infty$ (see (20) and (16)). The following lemma provides us with the spectral decomposition of $\tilde{\mathcal{L}}$ :

LEMMA 2.2 (Eigenvalues of $\mathcal{L}$ ).

i) $\mathcal{L}$ is a $\mathbb{R}$-linear operator defined on $L^{2}(\mathbb{R}, \mathbb{C}, d \mu)$ and its eigenvalues are given by $\left\{1-\frac{m}{2} \mid m \in \mathbb{N}\right\}$. Its eigenfunctions are given $b y$ $\left\{(1-\mid i \delta) h_{m}, i h_{m} \mid m \in \mathbb{N}\right\}$ where

$$
h_{m}(y)=\sum_{n=0}^{\left[\frac{m}{2}\right]} \frac{m !}{n !(m-2 n) !}(-1)^{n} y^{m-2 n} .
$$

We have: $\tilde{\mathcal{L}}\left((1+i \delta) h_{m}\right)=\left(1-\frac{m}{2}\right)(1+i \delta) h_{m}$ and $\tilde{\mathcal{L}}\left(i h_{m}\right)=-\frac{m}{2} i h_{m}$.

ii) Each $r \in L^{2}(\mathbb{R}, \mathbb{C}, d \mu)$ can be uniquely written as $r(y)=$ $(1+i \delta)\left(\sum_{m=0}^{+\infty} \hat{r}_{1, m} h_{m}(y)\right)+i\left(\sum_{m=0}^{+\infty} \hat{r}_{2, m} h_{m}(y)\right)$, where $\hat{r}_{j, m} \in \mathbb{R}$.

Proof.

i) From [18], we know that $\left\{h_{m} \mid m \in \mathbb{N}\right\}$ is a total family in $L^{2}(\mathbb{R}, \mathbb{R}, d \mu)$, and that $\left(\Delta-\frac{1}{2} y \cdot \nabla\right) h_{m}=-\frac{m}{2} h_{m}$. Hence, we decompose each $r \in L^{2}(\mathbb{R}, \mathbb{C}, d \mu)$ as $r(y)=\sum_{m=0}^{+\infty}\left(r_{1, m}+i r_{2, m}\right) h_{m}(y)$.

$\lambda \in \mathbb{R}$ is an eigenvalue for $\tilde{\mathcal{L}} \Longleftrightarrow \exists r \in L^{2}(\mathbb{R}, \mathbb{C}, d \mu), r \neq 0, \tilde{\mathcal{L}} r=\lambda r$

$\Longleftrightarrow \exists r \neq 0 \forall m \in \mathbb{N}\left\{\begin{aligned}\left(1-\frac{m}{2}-\lambda\right) & r_{1, m} \\ \lambda & r_{1, m}+\left(-\frac{m}{2}-\lambda\right) r_{2, m}=0\end{aligned}\right.$

$\Longleftrightarrow \exists m \in \mathbb{N} \lambda=1-\frac{m}{2}$. 
The computation of eigenfunctions is easy and we shall skip it.

ii) We write $r=(1+i \delta) \ddot{r}_{1}+i r_{2}$, with $\tilde{r}_{j} \in L^{2}(\mathbb{R}, \mathbb{R}, d \mu)$, and use the fact that $\left\{h_{m} \mid m \in \mathbb{N}\right\}$ is a total family in $L^{2}(\mathbb{R}, \mathbb{R}, d \mu)$.

Let us consider $(q(s), \theta(s))$ a solution of system (19). We will use an integral formulation of its first equation in terms of the fundamental solution of $\mathcal{L}_{\varphi}$. We want $\|q(s)\|_{L^{\infty}} \rightarrow 0$ as $s \rightarrow+\infty$. This $L^{\infty}$ control will result from the $L^{\infty}$ control of $(1-\chi(y, s)) q(y, s)$ and $\chi(y, s) q(y, s)$ (see (17) for $\chi$ ):

1 ) in the "regular" region $|y| \geq K_{0} \sqrt{s}, \mathcal{L}_{\varphi}$ behaves in $L^{2}(\mathbb{R}, \mathbb{C}, d \mu)$ like an operator with a fully negative spectrum. We will show from (20) that the fundamental solution of $\mathcal{L}_{\varphi}$ between $s_{0}$ and $s_{1}>s_{0}$ is a strict contraction from $L^{\infty}\left(|y| \geq K_{0} \sqrt{s}\right)$ to $L^{\infty}(\mathbb{R})$. Therefore, the control of $(1-\chi(y, s)) q(y, s)$ in $L^{\infty}(\mathbb{R})$ will be done without difficulties.

2 ) in the "singular" region $|y| \leq K_{0} \sqrt{s}, \mathcal{L}_{\varphi}$ behaves in $L^{2}(\mathbb{R}, \mathbb{C}, d \mu)$ like $\tilde{\mathcal{L}}$. In order to control $\chi q(y, s)$, we expand it with respect to the spectrum of $\tilde{\mathcal{L}}$ in $L^{2}(\mathbb{R}, \mathbb{C}, d \mu)$, but we will control $\chi q$ in $L^{\infty}(\mathbb{R})$ and not only in $L^{2}(\mathbb{R}, \mathbb{C}, d \mu)$ (see section 3 for the rigorous analysis).

By lemma $2.2, \tilde{\mathcal{L}}$ has two expanding directions $\left((1+i \delta) h_{0},(1+i \delta) h_{1}\right)$, two null ones $\left((1+i \delta) h_{2}, i h_{0}\right)$ and countably many negative ones.

Here, the situation is a bit more complicated than in [18], because we have two null directions (instead of only one).

Our strategy to control all the components of $\chi q$ so that $\|\chi q(s)\|_{L^{\infty}} \rightarrow 0$ as $s \rightarrow+\infty$ is to control the part of $\chi q$ corresponding to the negative spectrum of $\tilde{\mathcal{L}}$ and the one parallel to $(1+i \delta) h_{2}$ (which corresponds to the null eigenvalue) as in [18]. The component parallel to $i h_{0}$ (which corresponds also to the null eigenvalue) has been fixed by the second equation of (19) to be zero (using modulation theory and the phase invariance of the equation).

However, the analysis of system (19) is longer than the equivalent analysis in [18], because of terms with $\frac{d \theta}{d s}$, and the presence of strong coupling between the two scalar parts: $\ddot{q}_{1}$ and $\dot{q}_{2}$ of $q$, satisfying: $q=(1+i \delta) \ddot{q}_{1}+i \ddot{q}_{2}$. Fortunately, $\frac{d \theta}{d s}$ will be controlled near the profile $\varphi$ (see 16), and, although the coupling will be of critical size, its effect will be controlled by $\delta$, which can be chosen small.

\section{EXISTENCE OF A BLOW-UP SOLUTION FOR EQUATION (2)}

In this section, we prove proposition 2.1 , which implies parts i) and ii) of Proposition 1 and then Theorem 1.

Vol. 15. $n^{\circ}$ 5-1998. 


\subsection{Geometrical property for $q$}

As in [18], the convergence of $\|q(s)\|_{L^{x}}$ to zero as $s \rightarrow+\infty$ will follow from a geometrical property: $q(s) \in V_{A}(s)$, where $V_{A}(s) \subset L^{\infty}(\mathbb{R}, \mathbb{C})$ shrinks to $q \equiv 0$ as $s \rightarrow+\infty$. The structure of $V_{A}(s)$ respects the freeboundary moving in $q$ at the rate $\sqrt{s}$, and also the eigenfunctions of the operator $\tilde{\mathcal{L}}$ (Cf lemma 2.2).

In order to define $V_{A}(s)$, we introduce the following useful notations:

For each $g \in L^{\infty}(\mathbb{R}, \mathbb{R})$ and $s>0$, we define $g_{b}(y, s)=\chi(y, s) g(y)$ and $g_{e}(y, s)=(1-\chi(y, s)) g(y)$. Since $L^{\infty}(\mathbb{R}, \mathbb{R}) \subset L^{2}(\mathbb{R}, \mathbb{R}, d \mu)$, we introduce for each $m \in \mathbb{N}, g_{m}(s)$ as the $L^{2}(\mathbb{R}, \mathbb{R}, d \mu)$ projection of $g_{b}(y, s)$ on $h_{m}$, (Cf (21)). We also let $g_{-}(y, s)=P_{-}\left(g_{b}\right)$ and $g_{\perp}(y, s)=P_{\perp}\left(g_{b}\right)$, where $P_{-}$ and $P_{\perp}$ are the $L^{2}(\mathbb{R}, \mathbb{R}, d \mu)$ projectors respectively on Vect $\left\{h_{m} \mid m \geq 3\right\}$ and Vect $\left\{h_{m} \mid m \geq 1\right\}$. Thus, we write either

$$
g(y)=\sum_{m=0}^{2} g_{m}(s) h_{m}(y)+g_{-}(y, s)+g_{e}(y, s)
$$

or

$$
g(y)=g_{0}(s) h_{0}(y)+g_{\perp}(y, s)+g_{e}(y, s) .
$$

For each $z \in \mathbb{C}$, we write in a unique way $z=(1+i \delta) \tilde{z}_{1}+i \tilde{z}_{2}$, where $\tilde{z}_{1}$ and $\tilde{z}_{2}$ are real.

Hence, if $r \in L^{\infty}(\mathbb{R}, \mathbb{C})$, we write: $r(y)=(1+i \delta) \tilde{r}_{1}(y)+i \tilde{r}_{2}(y)$ and expand $\tilde{r}_{1}$ and $\tilde{r}_{2}$ respectively as in (22) and (23). Thus, we write: $r(y)=(1+i \delta) \tilde{r}_{1}(y)+i \tilde{r}_{2}(y)$

$$
\begin{aligned}
= & (1+i \delta)\left\{\sum_{m-0}^{2} \tilde{r}_{1, m}(s) h_{m}(y)+\tilde{r}_{1,-}(y, s)+\tilde{r}_{1, e}(y, s)\right\} \\
& +i\left\{\tilde{r}_{2,0}(s) h_{0}(y)+\tilde{r}_{2, \perp}(y, s)+\tilde{r}_{2, e}(y, s)\right\} .
\end{aligned}
$$

DEFINITION 3.1. - For each $A>0$, for each $s>0$, let $V_{A}(s)$ be the set of all functions $r$ in $L^{\infty}(\mathbb{R}, \mathbb{C})$ such that

$$
\begin{aligned}
& \left|\tilde{r}_{1, m}(s)\right| \leq A s^{-2} . \quad \text { for } m=0,1 \text {, } \\
& \left|\tilde{r}_{1,2}(s)\right| \leq A^{2}(\log s) s^{-2}, \quad\left|\tilde{r}_{2,0}(s)\right| \leq A s^{-2} . \\
& \left|\tilde{r}_{1,-}(y, s)\right| \leq A\left(1+|y|^{3}\right) s^{-2}, \quad\left|\tilde{r}_{2, \perp}(y, s)\right| \leq A\left(1+|y|^{3}\right) s^{-2} \text {, } \\
& \left\|\tilde{r}_{1, e}(s)\right\|_{L^{\infty}} \leq A^{2} s^{-\frac{1}{2}} . \quad\left\|\tilde{r}_{2, e}(s)\right\|_{L^{\infty}} \leq A^{2} s^{-\frac{1}{2}} \text {. }
\end{aligned}
$$

where $r$ is given by (24). 
Remark. - We note that $L^{\infty}(\mathbb{R}, \mathbb{C}) \subset L^{2}(\mathbb{R}, \mathbb{C}, d \mu)$, which justifies the expansion with respect to the eigenvalues of $\tilde{\mathcal{L}}$ in definition 3.1 .

Remark. - It is easy to see that if $q(s) \in V_{A}(s)$, then $\forall y \in \mathbb{R}$, $|q(y, s)| \leq C(A) s^{-1 / 2}$ (see [18] for details). Therefore, $\|q(s)\|_{L^{\infty}(\mathbb{R}, C)} \rightarrow 0$ as $s \rightarrow+\infty$, and we obtain a convergence in $L^{\infty}(\mathbb{R}, \mathbb{C})$ and not only in $L^{2}(\mathbb{R}, \mathbb{C}, d \mu)$, as in other papers (see $\left.[5], ..\right)$. We emphasize that a convergence in $L^{2}(\mathbb{R}, \mathbb{C}, d \mu)$ or more generally in $H^{m}(\mathbb{R}, \mathbb{C}, d \mu)$ yields a convergence in $L^{\infty}([-R, R], \mathbb{C})$ for each $R>0$, and never a uniform convergence on $\mathbb{R}$.

With this remark, we claim that proposition 2.1 follows from the following proposition:

Proposition 3.1 Equivalent formulation of proposition 1, i) and ii). There exists $A>0, \delta_{0}>0, S_{0}>0$, such that $\forall \delta \in\left[-\delta_{0}, \delta_{0}\right], \forall s_{0} \geq S_{0}$, $\exists\left(d_{0}, d_{1}\right) \in \mathbb{R}^{2}$ such that system (19) with initial data at $s=s_{0}$

$$
\left.\begin{array}{l}
q_{d_{0}, d_{1}}\left(y, s_{0}\right)=(1+i \delta) f_{0}\left(\frac{y}{\sqrt{s_{0}}}\right)^{p}\left(d_{0}+d_{1} y / \sqrt{s_{0}}\right)-\left(\frac{\alpha}{s_{0}}\right)^{1+i \delta} \\
+i \frac{\alpha}{s_{0}}\left(\sin \left[\delta \log \left(\frac{\alpha}{s_{0}}\right)\right]-\delta \cos \left[\delta \log \left(\frac{\alpha}{s_{0}}\right)\right]\right) f_{0}\left(\frac{y}{\sqrt{s_{0}}}\right)^{p} \beta\left(s_{0}\right) \\
\theta\left(s_{0}\right)=0
\end{array}\right\}
$$

(where $f_{0}$ is given by (6),

$$
\left.\alpha=\frac{\kappa}{2\left(p-\delta^{2}\right)}, \quad \beta\left(s_{0}\right)=\frac{\int f_{0}\left(\frac{y}{\sqrt{s_{0}}}\right)^{p} \chi\left(y, s_{0}\right) d \mu(y)}{\int \chi\left(y, s_{0}\right) d \mu(y)}\right)
$$

has a unique solution $(q, \theta)_{d_{0}, d_{1}}$ for $s \geq s_{0}$, satisfying $q(s) \in V_{A}(s)$, $\forall s \geq s_{0}$.

Indeed, once proposition 3.1 is proved, we take for $q_{s_{0}}$ the expression in (25). From $q(s) \in V_{A}(s), \forall s \geq s_{0}$, we have $\|q(s)\|_{L^{\infty}} \rightarrow 0$ as $s \rightarrow+\infty$, and $\exists \theta_{\infty}$ such that $\theta(s) \rightarrow \theta_{\infty}$ as $s \rightarrow+\infty$. Indeed, we have the following lemma:

LEMMA 3.1. - $\forall A>0, \exists s_{3}(A)>0$ such that $\forall \delta \in[-1,1], \forall s \geq s_{3}(A)$, if $q(s) \in V_{A}(s)$, then $\left|\frac{d \theta}{d s}(s)\right| \leq \frac{C}{s^{2}}$.

This lemma implies $\int_{s_{0}}^{+\infty}\left|\frac{d \theta}{d s}(s)\right| d s<+\infty$, which gives $\theta_{\infty}$ such that $\theta(s) \rightarrow \theta_{\infty}$ as $s \rightarrow+\infty$. We give the proof of this lemma in the next subsection. 
In order to understand the dynamics of $q$ and $\theta$, we derive the equations satisfied by $\tilde{q}_{1}$ and $\tilde{q}_{2}\left(q(y, s)-(1+i \delta) \tilde{q}_{1}(y, s)+i \tilde{q}_{2}(y, s)\right.$, Cf decomposition (24)) and $\theta$ :

Lemma 3.2 (Equations satisfied by $\tilde{q}_{1}, \tilde{q}_{2}$ and $\theta$ ). - If $q$ satisfies (19) for $s \geq s_{0}$, then:

$$
\begin{aligned}
& \frac{\partial \tilde{q}_{1}}{\partial s}(y, s)=\left(\mathcal{L}+V_{1,1}(y, s)+\delta \frac{d \theta}{d s}(s)\right) \tilde{q}_{1}+\left(V_{1,2}(y, s)+\frac{d \theta}{d s}(s)\right) \tilde{q}_{2} \\
&+\tilde{B}_{1}(q(y, s))+\tilde{R}_{1}(\theta, y, s) \\
& \frac{\partial \tilde{q}_{2}}{\partial s}(y, s)=\left(V_{2,1}-\left(1+\delta^{2}\right) \frac{d \theta}{d s}(s)\right) \tilde{q}_{1}+\left(\mathcal{L}-1+V_{2,2}(y, s)-\delta \frac{d \theta}{d s}(s)\right) \tilde{q}_{2} \\
&+ \tilde{B}_{2}(q(y, s))+\tilde{R}_{2}(\theta, y, s) \\
& \frac{d \theta}{d s} \int \chi(y, s)\left(\left(1+\delta^{2}\right) \tilde{\varphi}_{1}+\delta \tilde{\varphi}_{2}+\left(1+\delta^{2}\right) \tilde{q}_{1}+\delta \tilde{q}_{2}\right) d \mu \\
&=\int \chi(\mathcal{L}-1) \tilde{q}_{2} d \mu+\int \frac{\partial \chi}{\partial s} \tilde{q}_{2} d \mu+\int \chi\left(V_{2,1} \tilde{q}_{1}+V_{2,2} \tilde{q}_{2}\right) d \mu \\
&+\int \chi \tilde{B}_{2}(q) d \mu+\int \chi(y, s) \tilde{R}_{2}^{*}(y, s)
\end{aligned}
$$

where

$$
\begin{aligned}
& \text { where } \\
& \qquad \mathcal{L}=\Delta-\frac{1}{2} y \cdot \nabla+1 \\
& \left\{\begin{array}{l}
V_{1,1}(y, s)=\left(1-\delta^{2}\right)\left(|\varphi|^{p-1}-\frac{1}{p-1}\right)+(p-1)|\varphi|^{p-3}\left(\varphi_{1}^{2}-\delta^{2} \varphi_{2}^{2}\right)-1 \\
V_{1,2}(y, s)=-\delta\left(|\varphi|^{p-1}-\frac{1}{p-1}\right)+(p-1)|\varphi|^{p}{ }^{3}\left(\varphi_{1}-\delta \varphi_{2}\right) \varphi_{2} \\
V_{2,1}(y, s)=\left(1+\delta^{2}\right)\left\{\delta\left(|\varphi|^{p-1}-\frac{1}{p-1}\right)+(p-1)|\varphi|^{p-3}\left(\varphi_{1}+\delta \varphi_{2}\right) \varphi_{2}\right\} \\
V_{2,2}(y, s)=\left(1+\delta^{2}\right)\left\{\left(|\varphi|^{p-1}-\frac{1}{p-1}\right)+(p-1)|\varphi|^{p-3} \varphi_{2}^{2}\right\}
\end{array}\right.
\end{aligned}
$$

$\varphi$ is given by (16), $(1+i \delta) \tilde{B}_{1}+i \tilde{B}_{2}=B,(1+i \delta) \tilde{R}_{1}+i \tilde{R}_{2}=R$, and $B, R$ are given by (20).

Proof. - (27) and (28) follow directly from (19). For (29), we note that we derive form (19) $\frac{d}{d s} \int \chi(y, s) \tilde{q}_{2}(y, s) d \mu(y)=0\left(\tilde{q}_{2}=q_{2}-\delta q_{\perp}\right)$. Therefore $\int \chi(y, s) \frac{\partial \tilde{q}_{2}}{\partial s}(y, s) d \mu(y)=-\int \frac{\partial \chi}{\partial s}(y, s) \tilde{q}_{2}(y, s) d \mu(y)$. Multiplying (28) by $\chi$ and integrating with respect to $d \mu$ yields (29).

The proof of proposition 3.1 follows the general ideas developed in [18]. Indeed, it is divided in two parts:

- In a first part, we reduce the problem of the control in $V_{A}(s)$ of all the components of $q(s)$ to the problem of controlling $\left(\tilde{q}_{1,0}(s), \tilde{q}_{1,1}(s)\right)$, which 
are the components of $q$ corresponding to expanding directions of $\tilde{\mathcal{L}}$ (see (24) and lemma 2.2). That is, we reduce an infinite dimensional problem to a finite dimensional one.

- The second part of the proof is devoted to the solving of the finite dimensional problem, using 2-dimensional dynamics of $\left(\tilde{q}_{1,0}, \tilde{q}_{1,1}\right)(s)$ and a topological argument (index theory) based on the variation of the 2dimensional parameter $\left(d_{0}, d_{1}\right)$ appearing in the expression (25) of initial data $q_{d_{0}, d_{1}}\left(y, s_{0}\right)$.

\subsection{Proof of the geometrical property on $q(s)$}

First, we prove lemma 3.1 which insures that proposition 3.1 implies proposition 2.1 and then Proposition 1 i) and ii).

Proof of lemma 3.1. - We control $\frac{d \theta}{d s}$ thanks to equation (29). Let us estimate each term appearing in:

If $s_{0} \geq s_{3}(A)$, we have the following estimates.

- Since $q \in V_{A}$, the left-hand side of (29) is (in absolute value) greater than $C\left|\frac{d \theta}{d s}\right|$ where $C>0$.

- Since $\mathcal{L}$ is self-adjoint in $L^{2}\left(\mathbb{R}, d_{\mu}\right)$,

$\int \chi(\mathcal{L}-1) \tilde{q}_{2} d \mu=\int(\mathcal{L}-1) \chi \tilde{q}_{2} d \mu=\int\left(\frac{\partial^{2} \chi}{\partial y^{2}}-\frac{1}{2} y \frac{\partial \chi}{\partial y}\right) \tilde{q}_{2} e^{-y^{2} / 8} \frac{e^{-y^{2} / 8}}{\sqrt{4} \pi} d y$.

From (17), $\left|\frac{\partial^{2} \chi}{\partial y^{2}}-\frac{1}{2} y \frac{\partial \chi}{\partial y}\right| \leq C$, and $\frac{\partial^{2} \chi}{\partial y^{2}}-\frac{1}{2} y \frac{\partial \chi}{\partial y} \equiv 0$ for $|y| \leq K_{0} \sqrt{s}$. Hence, we can bound $e^{-y^{2} / 8}$ by $e^{-K_{0}^{2} s / 8}$, and use $q(s) \in V_{A}(s)$ to obtain $\left|\int \chi(\mathcal{L}-1) \tilde{q}_{2} d \mu\right| \leq C e^{-s}$ (if $K_{0}$ is large enough).

- The same argument yields $\left|\int \frac{\partial \chi}{\partial s} \tilde{q}_{2} d \mu\right| \leq C e^{-s}$.

- We have $\left|V_{i, j}(y, s)\right| \leq C s^{-1}\left(1+|y|^{2}\right)$ (see lemma B.1 in appendix B). Combining this with Definition 3.1, we get $\left|\int \chi\left(V_{2,1} \tilde{q}_{1}+V_{2,2} \tilde{q}_{2}\right) d \mu\right| \leq$ $C s^{-3} \log s$.

- We have $|\chi(y, s) B(q(y, s))| \leq C|q|^{2}$ for $q(s) \in V_{A}(s)$ (see lemma B.4). Therefore, $\left|\int \chi \tilde{B}_{2}(q) d \mu\right| \leq \int \chi|q|^{2} d \mu \leq C s^{-3}$.

- From (20), $\left|\int \chi(y, s) \tilde{R}_{2}^{*}(y, s)\right| \leq \frac{C}{s^{2}}$ (see lemma B.5).

Combining all the previous estimates gives: $\left|\frac{d \theta}{d s}\right| \leq \frac{C}{s^{2}}$.

Now, we give the proof of proposition 3.1 following the plan announced in the previous subsection.

PART I. - Reduction to a finite dimensional problem

Here, $(q, \theta)$ stands for a solution of system (19) with initial data (25). We show through a priori estimates that finding $\left(d_{0}, d_{1}\right) \in \mathbb{R}^{2}$ such that Vol. $15, n^{\circ} 5-1998$ 
$\forall s \geq s_{0} q(s) \in V_{A}(s)$ is equivalent to finding $\left(d_{0}, d_{1}\right) \in \mathbb{R}^{2}$ such that $\forall s \geq s_{0}\left(\tilde{q}_{1,0}(s), \tilde{q}_{1,1}(s)\right) \in \hat{V}_{A}(s)$, where

Definition 3.2. - For each $A>0$, for each $s>0$, we define $\hat{V}_{A}(s)$ as being the set $\left[-\frac{A}{s^{2}}, \frac{A}{s^{2}}\right]^{2} \subset \mathbb{R}^{2}$.

Proposition 3.2 (Control of $q(s)$ by $\left(\tilde{q}_{1,0}(s), \tilde{q}_{1,1}(s)\right.$ ) in $\tilde{V}_{A}(s)$ ). - There exists $A_{1}>0$ such that for each $A \geq A_{1}$, there exists $\delta_{1}(A)>0, s_{1}(A)>0$ such that for each $\delta \in\left[-\delta_{1}, \delta_{1}\right], s_{0} \geq s_{1}(A)$, we have the following properties:

- if $\left(d_{0}, d_{1}\right)$ is chosen so that $\left(\tilde{q}_{1,0}\left(s_{0}\right), \tilde{q}_{1,1}\left(s_{0}\right)\right) \in \hat{V}_{A}\left(s_{0}\right)$, and,

- if for $s_{1} \geq s_{0}$, we have $\forall s \in\left[s_{0}, s_{1}\right], q(s) \in V_{A}(s)$ and $q\left(s_{1}\right) \in$ $\partial V_{A}\left(s_{1}\right)$, then

i) $\left(\tilde{q}_{1,0}\left(s_{1}\right), \tilde{q}_{1,1}\left(s_{1}\right)\right) \in \partial \hat{V}_{A}\left(s_{1}\right)$,

ii) (transversality) there exists $\eta_{0}>0$ such that $\forall \eta \in\left(0, \eta_{0}\right)$, $\left(\tilde{q}_{1,0}\left(s_{1}+\eta\right), \tilde{q}_{1,1}\left(s_{1}+\eta\right)\right) \notin \hat{V}_{A}\left(s_{1}+\eta\right)$ (hence, $\left.q\left(s_{1}+\eta\right) \notin V_{A}\left(s_{1}+\eta\right)\right)$.

Proof. - see proof of proposition 3.2 below.

Now, we fix $A \geq A_{1}$, and $\delta_{0}=\delta_{1}$. We note $q\left(d_{0}, d_{1}\right)=q_{d_{0}, d_{1}}$ (see proposition 3.1).

PART II. - Topological argument for the finite dimensional problem

In the following proposition, we initialize the finite dimensional problem and study the Cauchy problem for system (19).

Proposition 3.3 (Initialization and Cauchy problem for system (19)). - There exists $s_{2}(A)>0$ such that for each $\delta \in\left[-\delta_{0}, \delta_{0}\right]$, for each $s_{0} \geq s_{2}(A)$,

i) there exists a set $\mathcal{D}_{s_{0}} \subset \mathbb{R}^{2}$ topologically equivalent to a square with the following property:

$$
q\left(d_{0}, d_{1}, s_{0}\right) \in V_{A}\left(s_{0}\right) \text { if and only if }\left(d_{0}, d_{1}\right) \in \mathcal{D}_{s_{0}} .
$$

ii) For each $\left(d_{0}, d_{1}\right) \in \mathcal{D}_{s_{0}}, \exists S=S\left(d_{0}, d_{1}\right)>s_{0}$ (maximal) such that system (19) with initial data (25) at $s=s_{0}$ has a unique solution $(q, \theta)\left(d_{0}, d_{1}\right)$ on $\left[s_{0}, S\right)$, with $q$ and $\theta \mathcal{C}^{2}$ and $q(s) \in V_{A+1}(s), \forall s \in\left[s_{0}, S\right)$.

iii) $(q, \theta)$ is continuous with respect to $\left(d_{0}, d_{1}, s\right)$.

Proof.

i) From (25), we have

$$
\tilde{q}_{1}\left(d_{0}, d_{1}, y, s_{0}\right)=f_{0}\left(\frac{y}{\sqrt{s_{0}}}\right)^{p}\left(d_{0}+d_{1} \frac{y}{\sqrt{s_{0}}}\right)-\frac{\alpha}{s_{0}} \cos \left[\delta \log \left(\frac{\alpha}{s_{0}}\right)\right]
$$


and

$$
\tilde{q}_{2}\left(d_{0}, d_{1}, y, s_{0}\right)=-\frac{\alpha}{s_{0}}\left(\delta-\sin \left[\delta \log \left(\frac{\alpha}{s_{0}}\right)\right]\right)\left(1-\beta\left(s_{0}\right) f_{0}\left(\frac{y}{\sqrt{s_{0}}}\right)^{p}\right) .
$$

The expression of $\tilde{q}_{1}$ is similar to the expression of initial data (31) for the similar equation (15) in [18]. $\tilde{q}_{2}$ is a sum of two terms appearing in the mentioned formula (31) in [18]. Hence, one can adapt without difficulties lemmas 3.5 and 3.9 of [18] to conclude (note that $\tilde{q}_{2,0}\left(d_{0}, d_{1}, s_{0}\right)=0$ ).

ii) As if to use (15) in a reverse way, we introduce

$$
w(y, s)=e^{i \theta(s)}(q(y, s)+\varphi(y, s)) .
$$

Therefore, our problem is equivalent to the following system in $(w, \theta)$ :

$$
\begin{gathered}
\frac{\partial w}{\partial s}=\Delta w-\frac{1}{2} y \cdot \nabla w-(1+i \delta) \frac{w}{p-1}+(1+i \delta)|w|^{p-1} w \\
F((\theta(s), s)=0
\end{gathered}
$$

where

$$
\begin{aligned}
F(\theta, s)= & \cos (\theta)\left(w_{2,0}(s)-\delta w_{1,0}(s)\right) \\
& +\sin (\theta)\left(-w_{1,0}(s)-\delta w_{2,0}(s)\right)-\tilde{\varphi}_{2,0}(s),
\end{aligned}
$$

with initial data

$$
\begin{aligned}
w\left(d_{0}, d_{1}, s_{0}\right) & =q\left(d_{0}, d_{1}, s_{0}\right)+\varphi\left(s_{0}\right), \\
\theta\left(s_{0}\right) & =0 .
\end{aligned}
$$

By a simple calculation, we have $w\left(d_{0}, d_{1}, s_{0}\right) \in H$. Hence from classical theory, we have local existence and uniqueness of a $\mathcal{C}^{2}$ solution for (32) with initial data (34).

In order to prove existence and uniqueness for $\theta(s)$, we apply the implicit function theorem to $F$ near $(\theta, s)=\left(0, s_{0}\right)$. First we compute $\frac{\partial F}{\partial \theta}(\theta, s)=-\sin (\theta)\left(w_{2,0}(s)-\delta w_{1,0}(s)\right)+\cos (\theta)\left(-w_{1,0}(s)-\delta w_{2,0}(s)\right)$ and $\frac{\partial F}{\partial \theta}\left(0, s_{0}\right)=-\varphi_{1,0}\left(s_{0}\right)-\delta \varphi_{2,0}\left(s_{0}\right)-\left(1+\delta^{2}\right) \ddot{q}_{1,0}\left(s_{0}\right)-\delta \ddot{q}_{2,0}\left(s_{0}\right)$ (use (31)). By (16), $-\varphi_{1,0}\left(s_{0}\right)-\delta \varphi_{2,0}\left(s_{0}\right) \rightarrow-\kappa$ as $s_{0} \rightarrow+\infty$. Hence, if $s_{0} \geq s_{2}(A)$ and $\left(d_{0}, d_{1}\right) \in \mathcal{D}_{s_{0}}$, then $q\left(s_{0}\right) \in V_{A}\left(s_{0}\right) \subset V_{A+1}\left(s_{0}\right)$ and $\frac{\partial F}{\partial \theta}\left(0, s_{0}\right) \neq 0$. Since $F\left(0, s_{0}\right)=0$ (because $\hat{q}_{2,0}\left(d_{0}, d_{1}, s_{0}\right)=0$ ), and $F$ is $\mathcal{C}^{2}$, we have existence and uniqueness of $\mathcal{C}^{2} \theta(s)$.

We add that the solution $(q, \theta)(s)$ is well defined if we require $q(s) \in V_{A+1}(s)$.

Vol. $15, n^{\circ} 5-1998$. 
iii) Using again the equivalent formulation (31), we see that $(q, \theta)\left(d_{0}, d_{1}, s\right)$ is a continuous function of $\left(q\left(d_{0}, d_{1}, s_{0}\right), s\right)$. Since $q\left(d_{0}, d_{1}, s_{0}\right)$ is continuous in $\left(d_{0}, d_{1}\right)$ (it is affine, see $(25)$ ), we obtain iii).

Now, we fix $S_{0}>\max \left(s_{1}(A), s_{2}(A)\right)$, and take $\delta \in\left[-\delta_{0}, \delta_{0}\right], s_{0} \geq S_{0}$. Then we start the proof of proposition 3.1 for $A, \delta$ and $s_{0}$.

We argue by contradiction: According to proposition 3.3, for each $\left(d_{0}, d_{1}\right) \in \mathcal{D}_{s_{0}}$, system (19) with initial data (25) has a unique solution on $\left[s_{0}, S\left(d_{0}, d_{1}\right)\right)$ and $q\left(d_{0}, d_{1}, s_{0}\right) \in V_{A}\left(s_{0}\right)$. We suppose then that for each $\left(d_{0}, d_{1}\right) \in \mathcal{D}_{s_{0}}$, there exists $s>s_{0}$ such that $q\left(d_{0}, d_{1}, s\right) \notin V_{A}(s)$. Let $s_{*}\left(d_{0}, d_{1}\right)$ be the infimum of all these $s$. By proposition $3.2\left(s_{1}=s_{*}\right)$, we can define the following function:

$$
\begin{aligned}
\Phi: \mathcal{D}_{s_{0}} & \longrightarrow \partial \mathcal{C} \\
\left(d_{0}, d_{1}\right) & \longrightarrow \frac{s_{*}\left(d_{0}, d_{1}\right)^{2}}{A}\left(\tilde{q}_{1,0}, \tilde{q}_{1,1}\right)\left(d_{0}, d_{1}, s_{*}\left(d_{0}, d_{1}\right)\right)
\end{aligned}
$$

where $\mathcal{C}$ is the unit square of $\mathbb{R}^{2}$.

Now we claim

Proposition 3.4. - i) $\Phi$ is a continuous mapping from $\mathcal{D}_{s_{0}}$ to $\partial \mathcal{C}$.

ii) There exists a non-trivial affine function $g: \mathcal{D}_{s_{0}} \rightarrow \mathcal{C}$ such that $\Phi \circ g_{\mid \partial \mathcal{C}}^{-1}=I d_{\mid \partial \mathcal{C}}$.

From that, a contradiction follows (Index Theory). Hence, there exists $\left(d_{0}, d_{1}\right)$ such that $\forall s \geq s_{0}, q\left(d_{0}, d_{1}, s\right) \in V_{A}(s)$.

This concludes the proof of proposition 3.1, and also the proof of parts i) and ii) of Proposition 1.

Proof of proposition 3.4:

i) Part iii) of proposition 3.3 implies that $\left(\tilde{q}_{1,0}(s), \tilde{q}_{1,1}(s)\right)$ is a continuous function of $\left(d_{0}, d_{1}\right)$. Using the transversality property of $\left(\tilde{q}_{1,0}\left(s_{*}\right), \tilde{q}_{1,1}\left(s_{*}\right)\right)$ on $\partial \hat{V}_{A}\left(s_{*}\right)$ ( ii) of proposition 3.2 ), we claim that $s_{*}\left(d_{0}, d_{1}\right)$ is continuous. Therefore, $\Phi$ is continuous.

ii) If $\left(d_{0}, d_{1}\right) \in \partial \mathcal{D}_{s_{0}}$, then from i) of proposition $3.3, q\left(d_{0}, d_{1}, s_{0}\right) \in$ $V_{A}\left(s_{0}\right)$. According to the proof of lemma 3.9 in [18], $\left(\tilde{q}_{1,0}\left(s_{0}\right), \tilde{q}_{1,1}\left(s_{0}\right)\right) \in$ $\partial \hat{V}_{A}\left(s_{0}\right)$. Applying ii) of proposition 3.2 with $s_{0}$ and $s_{1}=s_{0}$, we have $s_{*}\left(d_{0}, d_{1}\right)=s_{0}$, and $\Phi\left(d_{0}, d_{1}\right)=\frac{s_{0}^{2}}{A}\left(\tilde{q}_{1.0}\left(s_{0}\right), \tilde{q}_{1,1}\left(s_{0}\right)\right)$. Let $g$ : $\left.\left(d_{0}, d_{1}\right) \in \mathcal{D}_{s_{0}} \rightarrow \frac{s_{0}^{2}}{A}\left(\tilde{q}_{1,0}\left(s_{0}\right), \tilde{q}_{1,1}\right)\left(s_{0}\right)\right) \in \mathcal{C}$. From $(25), g$ is affine. Hence $\Phi \circ g_{\mid \partial \mathcal{D}_{s_{0}}}^{-1}=I d_{\mid \partial \mathcal{D}_{s_{0}}}$. This concludes the proof of proposition 3.4.

Now, we give the proof of proposition 3.2. 


\subsection{Proof of proposition 3.2}

As we suggested in the formulation of the problem, the proof follows the general ideas of [18]. However, it is more complicated because of terms with $\frac{d \theta}{d s}$ or because of strong interference between $\tilde{q}_{1}$ and $\tilde{q}_{2}$ (see (27), (28)). Therefore, we summarize arguments which are similar to those exposed in [18] by showing how to adapt them to the present context, and emphasize the arguments relative to these new terms.

We divide the proof in three steps:

- In Step 1, we give a priori estimates on $q(s)$ in $V_{A}(s)$ : assume that for given $A>0$ large, $\rho>0$ and an initial time $s_{0} \geq s_{4}(A, \rho)$, we have $q(s) \in V_{A}(s)$ for each $s \in[\sigma, \sigma+\rho]$, where $\sigma \geq s_{0}$. Using system (19) which is satisfied by $q$, we then derive new bounds on $\tilde{q}_{1,2}, \tilde{q}_{1,-}, \tilde{q}_{1, e}, \tilde{q}_{2, \perp}$ and $\tilde{q}_{2, e}$ in $[\sigma, \sigma+\rho]$ (involving $A$ and $\rho$ ).

- In Step 2, we show that these new bounds are better than those defining $V_{A}(s)$ (see definition 3.1 ) provided that $\rho \leq \rho^{*}(A)$. Since $\tilde{q}_{1,2}(s)=0$ by hypothesis in (19), only $\tilde{q}_{1,0}(s)$ and $\tilde{q}_{1,1}(s)$ remain to be controlled: the problem is then reduced to the control of a two dimensional variable $\left(\tilde{q}_{1,0}(s), \tilde{q}_{1,1}(s)\right)$. Afterwards, we conclude the proof of part i) of proposition 3.2 .

- In Step 3, we use dynamics of $\left(\tilde{q}_{1,0}(s), \tilde{q}_{1,1}(s)\right)$ to prove its transversality on $\partial V_{A}(s)$ (part ii) of proposition 3.2).

STEP 1. - A priori estimates of $q$.

From equations (27) and (28) (which are equivalent to the first equation of system (19)), we write the integral equations satisfied by $\tilde{q}_{1}$ and $\tilde{q}_{2}$ :

$$
\begin{aligned}
\tilde{q}_{1}(s)= & K_{1}(s, \sigma) \tilde{q}_{1}(\sigma)+\int_{\sigma}^{s} d \tau K_{1}(s, \tau) V_{1,2}(\tau) \tilde{q}_{2}(\tau) \\
& +\int_{\sigma}^{s} d \tau K_{1}(s, \tau) \tilde{B}_{1}(q) d \tau+\int_{\sigma}^{s} d \tau K_{1}(s, \tau) \tilde{R}_{1}^{*}(\tau) \\
& +\int_{\sigma}^{s} d \tau K_{1}(s, \tau) \frac{d \theta}{d s}(\tau)\left\{\delta \tilde{\varphi}_{1}(\tau)+\tilde{\varphi}_{2}(\tau)+\delta \tilde{q}_{1}(\tau)+\tilde{q}_{2}(\tau)\right\} \\
\tilde{q}_{2}(s)= & K_{2}(s, \sigma) \tilde{q}_{2}(\sigma)+\int_{\sigma}^{s} d \tau K_{2}(s, \tau) V_{2,1}(\tau) \tilde{q}_{1}(\tau) \\
& +\int_{\sigma}^{s} d \tau K_{2}(s, \tau) \tilde{B}_{2}(q) d \tau+\int_{\sigma}^{s} d \tau K_{2}(s, \tau) \tilde{R}_{2}^{*}(\tau) \\
& -\int_{\sigma}^{s} d \tau K_{2}(s, \tau) \frac{d \theta}{d s}(\tau) \\
& \left.\times\left\{\left(1+\delta^{2}\right) \tilde{\varphi}_{1}(\tau)+\delta \tilde{\varphi}_{2}(\tau)\right)+\left(1+\delta^{2}\right) \tilde{q}_{1}(\tau)+\delta \tilde{q}_{2}(\tau)\right\}
\end{aligned}
$$


where $K_{1}$ is the fundamental solution of $\mathcal{L}+V_{1,1}, K_{2}$ is the fundamental solution of $\mathcal{L}-1+V_{2,2}, \mathcal{L}$ is given by (30),

$B(q)=(1+i \delta) \tilde{B}_{1}+i \tilde{B}_{2}$, $R^{*}(y, s)-(1+i \delta) \tilde{R}_{1}^{*}+i \tilde{R}_{2}^{*}, B$ and $R^{*}$ are given by $(20)$.

We now assume that for each $s \in[\sigma, \sigma+\rho], q(s) \in V_{A}(s)$. Using $(36,37)$, we derive new bounds on all terms in the right hand sides of $(36,37)$, and then on $q$.

In the case $\sigma=s_{0}$, from initial data properties, it turns out that we obtain better estimates for $s \in\left[s_{0}, s_{0}+\rho\right]$.

More precisely, we have the following lemma:

Lemma 3.3. - There exists $A_{4}>0$ such that for each $A \geq A_{4}, \rho^{*}>0$, there exists $s_{4}\left(A, \rho^{*}\right)>0$ with the following property: $\forall \delta \in[-1 / 2,1 / 2]$, $\forall s_{0} \geq s_{4}\left(A, \rho^{*}\right), \forall \rho \leq \rho^{*}$, assume $\forall s \in[\sigma, \sigma+\rho], q(s) \in V_{A}(s)$ with $\sigma \geq s_{0}$

I) $\tilde{q}_{1}$ estimates:

We have $\forall s \in[\sigma, \sigma+\rho]$,

i) (main linear term)

$$
\begin{aligned}
\left|\alpha_{1,2}(s)\right| & \leq A^{2} \frac{\log \sigma}{s^{2}}+(s-\sigma) C A s^{-3} \\
\left|\alpha_{1,-}(y, s)\right| & \leq C\left(e^{-\frac{1}{2}(s-\sigma)} A+e^{-(s-\sigma)^{2}} A^{2}\right)\left(1+|y|^{3}\right) s^{-2}, \\
\left\|\alpha_{1, e}(s)\right\|_{L^{\infty}} & \leq C\left(A^{2} e^{-\frac{(s-\sigma)}{2 p}}+A e^{(s-\sigma)}\right) s^{-\frac{1}{2}}
\end{aligned}
$$

where, as in decomposition (22),

$$
K_{1}(s, \sigma) \tilde{q}_{1}(\sigma)=\alpha_{1}(y, s)=\sum_{m=0}^{2} \alpha_{1, m}(s) h_{m}(y)+\alpha_{1,-}(y, s)+\alpha_{1, e}(y, s) .
$$

If $\sigma=s_{0}$, and $q\left(s_{0}\right)$ satisfies (25), then

$$
\begin{aligned}
\left|\alpha_{1,2}(s)\right| & \leq \frac{\log s_{0}}{s^{2}}+C A\left(s-s_{0}\right) s^{-3} \\
\left|\alpha_{1,-}(y, s)\right| & \leq C\left(1+|y|^{3}\right) s^{-2}, \|\left.\alpha_{1, e}(s)\right|_{L^{\infty}} \leq C\left(1+e^{\left(s-s_{0}\right)}\right) s^{-\frac{1}{2}} .
\end{aligned}
$$

ii) (interference term)

$$
\begin{aligned}
\left|\iota_{1,2}(s)\right| & \leq C|\delta| A(s-\sigma) e^{s-\sigma} s^{-3}, \\
\left|\iota_{1,-}(y, s)\right| & \leq C|\delta| A^{2}(s-\sigma)\left(1+|y|^{3}\right) s^{-2}, \\
\left\|\iota_{1, e}(s)\right\|_{L^{\infty}} & \leq C|\delta|\left(A^{2}+e^{(s-\sigma)} A\right)(s-\sigma) s^{-1 / 2},
\end{aligned}
$$


where, as in decomposition (22), $\int_{\sigma}^{s} d \tau K_{1}(s, \tau) V_{1,2}(\tau) \tilde{q}_{2}(\tau)=$

$$
\iota_{1}(y, s)=\sum_{m=0}^{2} \iota_{1, m}(s) h_{m}(y)+\iota_{1,-}(y, s)+\iota_{1, e}(y, s)
$$

iii) (nonlinear term)

$$
\begin{aligned}
\left|\beta_{1,2}(s)\right| & \leq \frac{(s-\sigma)}{s^{3+1 / 2}} \\
\left|\beta_{1,-}(y, s)\right| & \leq(s-\sigma)\left(1+|y|^{3}\right) s^{-2-\varepsilon},\left\|\beta_{1, e}(s)\right\|_{L^{\infty}} \leq(s-\sigma) s^{-\frac{1}{2}-\varepsilon}
\end{aligned}
$$

where $\varepsilon=\varepsilon(p)>0$, and as in $(22), \int_{\sigma}^{s} d \tau K_{1}(s, \tau) \tilde{B}_{1}(q(\tau))=$

$$
\beta_{1}(y, s)=\sum_{m=0}^{2} \beta_{1, m}(s) h_{m}(y)+\beta_{1,-}(y, s)+\beta_{1, e}(y, s) .
$$

iv) (main corrective term)

$$
\begin{aligned}
\left|\gamma_{1,2}(s)\right| & \leq(s-\sigma) C s^{-3} \\
\left|\gamma_{1,-}(y, s)\right| & \leq(s-\sigma) C\left(1+|y|^{3}\right) s^{-2},\left\|\gamma_{1, e}(s)\right\|_{L^{\infty}} \leq(s-\sigma) s^{-3 / 4},
\end{aligned}
$$

where as in (22),

$\int_{\sigma}^{s} d \tau K_{1}(s, \tau) \tilde{R}_{1}^{*}(\tau)=\gamma_{1}(y, s)=\sum_{m=0}^{2} \gamma_{1, m}(s) h_{m}(y)+\gamma_{1,-}(y, s)+\gamma_{1, e}(y, s)$

v) (small terms)

$$
\begin{aligned}
\left|\lambda_{1,2}(s)\right| & \leq C(s-\sigma) s^{-3}, \\
\left|\lambda_{1,-}(y, s)\right| & \leq C(s-\sigma)\left(1+|y|^{3}\right) s^{-3},\left\|\lambda_{1, e}(s)\right\|_{L^{\infty}} \leq C(s-\sigma) s^{-3 / 2},
\end{aligned}
$$

where as in $(22), \int_{\sigma}^{s} d \tau K_{1}(s, \tau) \frac{d \theta}{d s}(\tau)\left\{\delta \tilde{q}_{1}(\tau)+\tilde{q}_{2}(\tau)+\delta \tilde{\varphi}_{1}(\tau)+\tilde{\varphi}_{2}(\tau)\right\}=$

$$
\lambda_{1}(y, s)=\sum_{m=0}^{2} \lambda_{1, m}(s) h_{m}(y)+\lambda_{1,-}(y, s)+\lambda_{1, e}(y, s) .
$$

II) $\tilde{q}_{2}$ estimates:

We have $\forall s \in[\sigma, \sigma+\rho]$,

i) (main linear term)

$$
\begin{aligned}
\left|\alpha_{2, \perp}(y, s)\right| & \leq C\left(e^{-\frac{1}{2}(s-\sigma)} A+e^{-(s-\sigma)^{2}} A^{2}\right)\left(1+|y|^{3}\right) s^{-2} \\
\left\|\alpha_{2, e}(s)\right\|_{L^{\infty}} & \leq C\left(A^{2} e^{-\frac{(s-\sigma)}{P}}+A\right) s^{-\frac{1}{2}}
\end{aligned}
$$


where, as in decomposition (23),

$$
K_{2}(s, \sigma) \tilde{q}_{2}(\sigma)=\alpha_{2}(y, s)=\alpha_{2,0}(s) h_{0}(y)+\alpha_{2, \perp}(y, s)+\alpha_{2, e}(y, s)
$$

If $\sigma=s_{0}$, and $q\left(s_{0}\right)$ satisfies (25), then

$$
\left|\alpha_{2, \perp}(y, s)\right| \leq C\left(1+|y|^{3}\right) s^{-2},\left\|\alpha_{2, e}(s)\right\|_{L^{\infty}} \leq C s^{-\frac{1}{2}}
$$

\section{ii) (interference term)}

$\left|\iota_{2, \perp}(y, s)\right| \leq C|\delta| A(s-\sigma)\left(1+|y|^{3}\right) s^{-2},\left\|\iota_{2, e}(s)\right\|_{L^{\infty}} \leq C|\delta| A^{2}(s-\sigma) s^{-1 / 2}$, where as in (23),

$$
\begin{gathered}
\int_{\sigma}^{s} d \tau K_{2}(s, \tau) V_{2,1}(\tau) \tilde{q}_{1}(\tau)= \\
\iota_{2}(y, s)=\iota_{2,0}(s) h_{0}(y)+\iota_{2, \perp}(y, s)+\iota_{2, e}(y, s) .
\end{gathered}
$$

iii) (nonlinear term)

$$
\left|\beta_{2, \perp}(y, s)\right| \leq(s-\sigma)\left(1+|y|^{3}\right) s^{-2-\varepsilon},\left\|\beta_{2, e}(s)\right\|_{L^{\infty}} \leq(s-\sigma) s^{-\frac{1}{2}-\varepsilon},
$$

where $\varepsilon=\varepsilon(p)>0$, and as in (23),

$$
\int_{\sigma}^{s} d \tau K_{2}(s, \tau) \tilde{B}_{2}(q(\tau))=\beta_{2}(y, s)=\beta_{2,0}(s) h_{0}(y)+\beta_{2, \perp}(y, s)+\beta_{2, e}(y, s) .
$$

iv) (main corrective term)

$$
\left|\gamma_{2, \perp}(y, s)\right| \leq C s^{-2}(s-\sigma)\left(1+|y|^{3}\right),\left\|\gamma_{2, e}(s)\right\|_{L^{\infty}} \leq(s-\sigma) s^{-3 / 4},
$$

where as in (23),

$$
\int_{\sigma}^{s} d \tau K_{2}(s, \tau) \tilde{R}_{2}^{*}(\tau)=\gamma_{2}(y, s)=\gamma_{2,0}(s) h_{m}(y)+\gamma_{2, \perp}(y, s)+\gamma_{2, e}(y, s)
$$

\section{v) (small terms)}

$$
\left|\lambda_{2, \perp}(y, s)\right| \leq C(s-\sigma)\left(1+|y|^{3}\right) s^{-2},\left\|\lambda_{2, e}(s)\right\|_{L^{\infty}} \leq C(s-\sigma) s^{-2},
$$

where $\int_{\sigma}^{s} d \tau K_{2}(s, \tau) \frac{d \theta}{d s}(\tau)\left\{-\delta \tilde{q}_{2}(\tau)-\left(1+\delta^{2}\right) \tilde{q}_{1}(\tau)-\delta \tilde{\varphi}_{2}(\tau)-(1+\right.$ $\left.\left.\delta^{2}\right) \tilde{\varphi}_{1}(\tau)\right\}=\lambda_{2}(y, s)=\lambda_{2,0}(s) h_{0}(y)+\lambda_{2, \perp}(y, s)+\lambda_{2, e}(y, s)$, as in (23). 


\section{Proof. - see appendix B.}

STEP 2. - Lemma 3.3 implies i) of proposition 3.2.

Here, we derive i) of proposition 3.2 from lemma 3.3. We follow the method used in [18] to prove proposition 3.11 starting from lemma 3.12 . Indeed, from integral equations $(36,37)$ and lemma 3.3 , we derive new bounds on $\tilde{q}_{1,2}(s), \tilde{q}_{1,-}(y, s), \tilde{q}_{1, e}(y, s), \tilde{q}_{2, \perp}(s)$ and $\tilde{q}_{2, e}(y, s)$, assuming that $\forall s \in[\sigma, \sigma+\rho], q(s) \in V_{A}(s)$, for $\rho \leq \rho^{*}$ and $\sigma \geq s_{0} \geq s_{4}\left(A, \rho^{*}\right)$. The key estimate is to show that for $s=\sigma+\rho$ (or $s \in[\sigma, \sigma+\rho]$ if $\sigma=s_{0}$ ), these new bounds are better than those defining $V_{A}(s)$, provided that $\rho \leq \rho^{*}(A)$.

Comparing lemma 3.3 here and lemma 3.12 in [18], we see that we have additional terms:

- Interference terms I ii) and II ii),

- Small terms I v) and II v).

If we try to adapt the proof of proposition 3.11 of [18] in order to prove a similar result, we see that the introduction of small terms does not change anything to the proof, since they are either of lower order, if compared for example with linear terms (speaking in terms of power of $s$ ): $\lambda_{1 .-}, \lambda_{1, e}$ and $\lambda_{2, e}$, or of the same order, but with a "small" coefficient (compared with $A$ ): $\lambda_{1,2}$ and $\lambda_{2, \perp}$.

This is not the case of interference terms Ii) and II i), which have a critical growth in terms of power of $s$. But recalling that in the mentioned proof in [18], we have $(s-\sigma) \leq \rho \leq \rho^{*} \leq \log \frac{A}{C^{*}}$, if we assume that:

$C|\delta| A \log \frac{A}{C^{*}} e^{\log \frac{A}{C^{*}}} \leq 1$ (Cf $\left.\iota_{1,2}\right), C|\delta| A^{2} \log \frac{A}{C^{*}} \leq \frac{A}{4}$ (Cf $\iota_{1,-}$ ), $C|\delta|\left(A^{2}+e^{\log \frac{A}{C^{*}}} A\right) \log \frac{A}{C^{*}} \leq \frac{A^{2}}{4}\left(\mathrm{Cf} \iota_{1, e}\right), C|\delta| A \log \frac{A}{C^{*}} \leq \frac{A}{4}\left(\mathrm{Cf} \iota_{2, \perp}\right)$ and $C|\delta| A^{2} \log \frac{A}{C^{*}} \leq \frac{A^{2}}{4}\left(\right.$ Cf $\left.\iota_{2, e}\right)$,

which is possible if $|\delta| \leq \delta_{5}(A)$, with $\delta_{5}(A)>0$, then all these terms, while remaining with critical growth, have a reasonable coefficient $\left(1, \frac{A}{4}\right.$ or $\left.\frac{A^{2}}{4}\right)$.

Therefore, adapting the proof of proposition 3.11 in [18] for $|\delta| \leq \delta_{5}(A)$, we prove a similar proposition:

Proposition 3.5. - There exists $A_{5}>0$ such that for each $A \geq A_{5}$, there exists $\delta_{5}(A)>0, s_{5}(A)>0$ such that for each $\delta \in\left[-\delta_{5}, \delta_{5}\right], s_{0} \geq s_{5}(A)$, we have the following property:

- if $\left(d_{0}, d_{1}\right)$ is chosen so that $\left(\tilde{q}_{1,0}\left(s_{0}\right), \tilde{q}_{1,1}\left(s_{0}\right)\right) \in \hat{V}_{A}\left(s_{0}\right)$, and,

- if for $s_{1} \geq s_{0}$, we have $\forall s \in\left[s_{0}, s_{1}\right], q(s) \in V_{A}(s)$,

then $\forall s \in\left[s_{0}, s_{1}\right],\left|\tilde{q}_{1,2}(s)\right| \leq A^{2} s^{-2} \log s-s^{-3},\left|\tilde{q}_{1,-}(y, s)\right| \leq$ $\frac{A}{2}\left(1+|y|^{3}\right) s^{-2},\left\|\tilde{q}_{1, e}(s)\right\|_{L^{\infty}} \leq \frac{A^{2}}{2 \sqrt{g}}, \quad\left|\tilde{q}_{2, \perp}(y, s)\right| \leq \frac{A}{2}\left(1+|y|^{3}\right) s^{-2}$, $\left\|\tilde{q}_{2, e}(s)\right\|_{L^{\infty}} \leq \frac{A^{2}}{2 \sqrt{s}}$. 
By definition of $(q, \theta)$ (Cf system (19)), we have $\tilde{q}_{2,0}(s)=0$. If in addition $q\left(s_{1}\right) \in \partial V_{A}\left(s_{1}\right)$, we see from definition 3.1 of $V_{A}(s)$ that the first two components of $q\left(s_{1}\right)$, namely $\tilde{q}_{1,0}\left(s_{1}\right)$ and $\tilde{q}_{1,1}\left(s_{1}\right)$ are in $\partial \hat{V}_{A}\left(s_{1}\right)$. This concludes the proof of part i) of proposition 3.2 .

STEP 3. - Transversality property of $\left(\tilde{q}_{1,0}\left(s_{1}\right), \tilde{q}_{1,1}\left(s_{1}\right)\right)$ on $\partial \hat{V}_{A}\left(s_{1}\right)$.

To prove part ii) of proposition 3.2, we show that for each $m \in\{0,1\}$, for each $\varepsilon \in\{-1,1\}$, if $\tilde{q}_{1, m}\left(s_{1}\right)=\varepsilon \frac{A}{s_{1}^{2}}$, then $\frac{d \tilde{q}_{1, m}}{d s}\left(s_{1}\right)$ has the opposite sign of $\frac{d}{d s}\left(\frac{\varepsilon A}{s^{2}}\right)\left(s_{1}\right)$ so that $\left(\tilde{q}_{1,0}, \tilde{q}_{1,1}\right)$ actually leaves $\hat{V}_{A}$ at $s_{1}$ for $s_{1} \geq s_{0}$ where $s_{0}$ will be large. Now, let us compute $\frac{d \tilde{q}_{1,0}}{d s}\left(s_{1}\right)$ and $\frac{d \tilde{q}_{1,1}}{d s}\left(s_{1}\right)$ for $q\left(s_{1}\right) \in V_{A}\left(s_{1}\right)$ and $\left(\tilde{q}_{1,0}\left(s_{1}\right), \tilde{q}_{1,1}\left(s_{1}\right)\right) \in \partial \hat{V}_{A}\left(s_{1}\right)$. First, we note that in this case, $\left\|q\left(s_{1}\right)\right\|_{L^{\infty}} \leq \frac{C A^{2}}{\sqrt{s_{1}}}$ and $\left|q_{b}\left(y, s_{1}\right)\right| \leq C A^{2} \frac{\log s_{1}}{s_{1}^{2}}\left(1+|y|^{3}\right)$ (Provided $A \geq 1$ ). Below, $O(l)$ stands for a quantity whose absolute value is bounded precisely by $l$ and not $C l$.

For $m \in\{0,1\}$, we derive from equation (27) and (21):

$$
\begin{gathered}
\int d \mu \chi\left(s_{1}\right) \frac{\partial \tilde{q}_{1}}{\partial s} k_{m}= \\
\int d \mu \chi\left(s_{1}\right) \mathcal{L} \tilde{q}_{1} k_{m}+\int d \mu \chi\left(s_{1}\right)\left\{V_{1,1} \tilde{q}_{1}+V_{1,2} \tilde{q}_{2}\right\} k_{m}+\int d \mu \chi\left(s_{1}\right) \tilde{B}_{1}(q) k_{m} \\
+\int d \mu \chi\left(s_{1}\right) \tilde{R}_{1}^{*}\left(s_{1}\right) k_{m}+\int d \mu \chi\left(s_{1}\right) \frac{d \theta}{d s}\left(s_{1}\right)\left\{\delta \tilde{q}_{1}+\tilde{q}_{2}+\delta \tilde{\varphi}_{1}+\tilde{\varphi}_{2}\right\} k_{m},
\end{gathered}
$$

where $k_{m}=h_{m} /\left\|h_{m}\right\|_{L^{2}(\mathbb{R}, \mathbb{R}, d \mu)}^{2}$ (see (21)).

We now estimate each term of this identity:

a) $\left|\int d \mu \chi\left(s_{1}\right) \frac{\partial \tilde{q}_{1}}{\partial s} k_{m}-\frac{d \tilde{q}_{1, m}}{d s}\right|=\left|\int d \mu \frac{d \chi}{d s} \tilde{q}_{1} k_{m}\right| \leq \int d \mu\left|\frac{d \chi}{d s}\right| \frac{C A^{2}}{\sqrt{s_{1}}}\left|k_{m}\right| \leq$ $C e^{-s_{1}}$ if $s_{0} \geq s_{3}(A)$.

b) Since $\mathcal{L}$ is self-adjoint on $L^{2}(\mathbb{R}, d \mu)$, we write

$$
\int d \mu \chi\left(s_{1}\right) \mathcal{L} \tilde{q}_{1} k_{m}-\int d \mu \mathcal{L}\left(\chi\left(s_{1}\right) k_{m}\right) \tilde{q}_{1} .
$$

Using $\mathcal{L}\left(\chi\left(s_{1}\right) k_{m}\right)-\left(1-\frac{m}{2}\right) \chi\left(s_{1}\right) k_{m}+\frac{\partial^{2} \chi}{\partial s^{2}} k_{m}+\frac{\partial \chi}{\partial y}\left(2 \frac{\partial k_{m}}{\partial y}-\frac{y}{2} k_{m}\right)$, we obtain $\int d \mu \chi\left(s_{1}\right) \mathcal{L} \tilde{q}_{1} k_{m}=\left(1-\frac{m}{2}\right) \tilde{q}_{1, m}\left(s_{1}\right)+O\left(C A e^{-s_{1}}\right)$.

c) We have $\forall y \in \mathbb{R},\left|V_{i, j}(y, s)\right| \leq \frac{C}{s}\left(1+|y|^{2}\right)$. Therefore, $\left|\int d \mu \chi\left(s_{1}\right)\left\{V_{1,1} \tilde{q}_{1}+V_{1,2} \tilde{q}_{2}\right\} k_{m}\right| \leq \int d \mu C s_{1}^{-1}\left(1+|y|^{2}\right) C A^{2} s_{1}^{-2} \log s_{1}\left|k_{m}\right|$ $\leq C A^{2} s_{1}^{-3} \log s_{1}$

d) A standard Taylor expansion combined with the definition of $V_{A}$ shows that $\left|\chi\left(y, s_{1}\right) B\left(q\left(y, s_{1}\right)\right)\right| \leq C|q|^{2} \leq C\left(\left|q_{b}\right|^{2}+\left|q_{e}\right|^{2}\right) \leq$ 
$\frac{C A^{4}\left(\log s_{1}\right)^{2}}{s_{1}^{4}}\left(1+|y|^{3}\right)^{2}+1_{\left\{|y| \geq K \sqrt{s}_{1}\right\}}(y) \frac{A^{2}}{\sqrt{s}_{1}}$. Thus, $\left|\int d \mu \chi\left(s_{1}\right) \tilde{B}_{1}(q) k_{m}\right| \leq$ $\frac{C A^{4}\left(\log s_{1}\right)^{2}}{s_{1}^{4}}+C e^{-s_{1}}$.

e) From lemma B.5 in appendix B, we have $\left|\int d \mu \chi\left(s_{1}\right) \tilde{R}_{1}^{*}\left(s_{1}\right) k_{m}\right| \leq$ $\frac{C(p)}{s_{1}^{2}}$ (Actually it is equal to 0 if $m=1$ ).

f) From lemma 3.1, we have $\left|\frac{d \theta}{d s}\left(s_{1}\right)\right| \leq C s_{1}^{-2}$. Hence, $\left|\int d \mu \chi\left(s_{1}\right) \frac{d \theta}{d s}\left(s_{1}\right)\left\{\delta \tilde{q}_{1}+\tilde{q}_{2}+\delta \tilde{\varphi}_{1}+\tilde{\varphi}_{2}\right) k_{m}\right| \leq C s_{1}^{-2}$.

Putting together the estimates a) to $f$ ), we obtain

$$
\frac{d \tilde{q}_{1, m}}{d s}\left(s_{1}\right)=\left(1-\frac{m}{2}\right) \frac{\varepsilon A}{s_{1}^{2}}+O\left(\frac{C(p)}{s_{1}^{2}}\right)+O\left(C A^{4} \frac{\log s_{1}}{s_{1}^{3}}\right)
$$

whenever $\tilde{q}_{1, m}\left(s_{1}\right)=\frac{\varepsilon A}{s_{1}^{2}}$. Let us now fix $A \geq 2 C(p)$, and then we take $s_{1}(A)$ larger so that for $s_{0} \geq s_{1}(A), \forall s \geq s_{0}, \frac{C(p)}{s^{2}}+O\left(C A^{4} \frac{\log s}{s^{3}}\right) \leq \frac{3 C(p)}{2 s^{2}}$. Hence, if $\varepsilon=-1, \frac{d \tilde{q}_{1, m}}{d s}\left(s_{1}\right)<0$, if $\varepsilon=1, \frac{d \tilde{q}_{1, m}}{d s}\left(s_{1}\right)>0$. This concludes the proof of part ii) of proposition 3.2. It also concludes the proof of part ii) of proposition 1, and then the proof of Theorem 1.

\section{BLOW-UP PROFILE OF $u(t)$ SOLUTION OF (2) NEAR BLOW-UP POINT}

We prove in this section part iii) of Proposition 1.

We consider $u(t)$ solution of (1) constructed in section 3, which blowsup in finite time $T>0$ at only one blow-up point: 0 . We know from section 3 that:

$$
\sup _{z \in \mathbb{R}}\left|(T-t)^{\frac{1+i \delta}{p-1}} u(z \sqrt{(T-t)|\log (T-t)|}, t)-f(z)\right| \leq \frac{C}{\sqrt{|\log (T-t)|}}
$$

with

$$
f(z)=\left(p-1+\frac{(p-1)^{2}}{4\left(p-\delta^{2}\right)}|z|^{2}\right)^{-\frac{1+i \delta}{p-1}} .
$$

Adapting the techniques used by Merle in [17] to equation (1), we derive the existence of a profile $u_{*} \in \mathcal{C}(\mathbb{R} \backslash\{0\}, \mathbb{C})$ such that $u(x, t) \rightarrow u_{*}(x)$ as $t \rightarrow T$ uniformly on compact subsets of $\mathbb{R} \backslash\{0\}$. We want to find an equivalent function for $u_{*}$ near the blow-up point: 0 .

For this purpose, we define for each $t \in[0, T)$, a rescaled version of $u(t)$ :

$$
v(t, \xi, \tau)=(T-t)^{\frac{1+i b}{p-1}} u(\xi \sqrt{T-t}, t+(T-t) \tau)
$$


where $\xi \in \mathbb{R}, \tau \in\left[-\frac{t}{T-t}, 1\right) \subset[0,1)$. From equation (1), we see that $v(t, \xi, \tau)$ satisfies the same equation as $u(t, x)$ :

$$
\forall \tau \in\left[-\frac{t}{T-t}, 1\right), \frac{\partial v}{\partial \tau}-\Delta_{\xi} v+(1+i \delta)|v|^{p-1} v .
$$

Stated in terms of $v(t)$. (39) becomes:

$$
\begin{gathered}
\sup _{\xi \in \mathbb{R}}\left|(1-\tau)^{\frac{1+i \delta}{p-1}} v(t, \xi, \tau)-f\left(\frac{\xi}{\sqrt{(1-\tau)|\log \{(1-\tau)(T-t)\}|}}\right)\right| \\
\leq \frac{C}{\sqrt{|\log \{(T-t)(1-\tau)\}|}}
\end{gathered}
$$

We proceed in two steps:

- first, we consider $r>0$ and estimate $v(t, \xi, \tau)$ and its derivatives locally near $\xi(r, t) \in \mathbb{R}$ satisfying $|\xi(r, t)|=r \sqrt{|\log (T-t)|}$. We show that $v(t, \xi, \tau)$ is bounded, and that it does not vary much for $|\xi-\xi(r, t)|$ bounded and $\tau \in[0,1]$,

- then, we can identify $v(t, \xi, 0)$ (approximated by (43)) and $v(t, \xi, 1)$. For each $x \in \mathbb{R} \backslash\{0\}$, we write $|x|$ as $|\xi(r, t)| \sqrt{(T-t)}=$ $r \sqrt{(T-t)|\log (T-t)|}$ for some $r>0$ and $t<T$ and combine this identification with (41) to get the equivalent of $u_{*}(x)$ for $x \rightarrow 0$ :

$$
u_{*}(x) \sim\left[\frac{8\left(p-\delta^{2}\right)|\log | x||}{(p-1)^{2}|x|^{2}}\right]^{\frac{1+i \delta}{p-1}} .
$$

For simplicity, we omit $t$ in the notation and write $v(\xi, \tau)$ for $v(t, \xi, \tau)$, $\xi(r)$ for $\xi(r, t)$.

PART I. - Estimate for $v$ near $r \sqrt{|\log (T-t)|}$.

From (41), $v$ blows-up at time $\tau=1$ at only one blow-up point: 0 . Using (43) and a lower bound shown by Giga and Kohn in [11] on blow-up rate for $v$, we derive a local bound on $v$ for $\tau \in[0,1),|\xi-\xi(r)|$ bounded, independent from $r$ and $t$. Using classical parabolic theory and the fact that $v$ depends in a certain sense only on $\tau$ for $|\tau|$ small, we show that $v$ actually does not depend much on $\tau \in[0,1)$ for $|\xi-\xi(r)|$ bounded.

Proposition 4.1 Estimate on $\frac{\partial v}{\partial \tau}(\xi(r), \tau)$. - There exists $r_{1}>0$ such that $\forall r \geq r_{1}, \exists t_{1}(r)<T$ such that $\forall t \in\left[t_{1}(r), T\right), \forall \tau \in[0,1)$, $\left|\frac{\partial v}{\partial \tau}(\xi(r), \tau)\right| \leq C|f(r)|^{p}$.

STEP 1. - Local bounds on $v$ near $\xi(r)$ for $\tau \in[-1 / 2,1)$. 
We crucially use a lower bound on blow-up rate for $v$ established by Giga and Kohn in [11] to show that $|v|$ is bounded for $\xi$ near $\xi(r)$ and $\tau \in[-1 / 2,1)$.

LEMMA 4.1 (Lower bound on blow-up rate for $v$ )

i) (Giga-Kohn) There exists $\varepsilon=\varepsilon(p, \delta, N)>0$ with the following property: If for $|\xi-\xi(r)| \leq 3 \sqrt{|\log (T-t)|}, \tau \in[-1 / 2,1)$

$$
(1-\tau)^{\frac{1}{p-1}}|v(\xi, \tau)| \leq \varepsilon,
$$

then $\forall \xi \in \mathbb{R}$ with $|\xi-\xi(r)| \leq 2 \sqrt{|\log (T-t)|}, \quad \forall \tau \in[-1 / 2,1)$, $|v(\xi, \tau)| \leq C$.

ii) There exists $r_{2}>0$ such that $\forall r \geq r_{2}, \exists t_{2}(r)<T$ such that $\forall t \in\left[t_{2}(r), T\right)$, if $|\xi-\xi(r)| \leq 2 \sqrt{|\log (T-t)|}, \tau \in[-1 / 2,1)$ then

$$
|v(\xi, \tau)| \leq C .
$$

\section{Proof.}

i) follows immediately from Theorem 2.1 in [11]. ii) is a direct consequence of i) and estimate (43). Indeed, if $|\xi-\xi(r)| \leq 3 \sqrt{|\log (T-t)|}$ and $\tau \in[-1 / 2,1)$, then we have by (43) $(1-\tau)^{\frac{1}{p-1}}|v(\xi, \tau)| \leq$ $C|f(r)|+C|\log (T-t)|^{-1 / 2}$.

STEP 2. - Local bound on $\frac{\partial v}{\partial \tau}(\xi, \tau)$ near $\xi(r)$ for $\tau \in[0,1)$

$-\tau=0$ : From a parabolic estimate and (43) considered for $\tau \leq 0$, we have for $|\xi-\xi(r)| \leq \sqrt{|\log (T-t)|}$ :

$$
\left|\frac{\partial^{2} v}{\partial \xi^{2}}(\xi, 0)-\frac{1}{|\log (T-t)|} \frac{\partial^{2} f}{\partial z^{2}}\left(\frac{\xi}{\sqrt{|\log (T-t)|}}\right)\right| \leq \frac{C}{\sqrt{|\log (T-t)|}} .
$$

Hence, from (42), we have for $r \geq r_{3}, t \geq t_{3}(r),|\xi-\xi(r)| \leq$ $\sqrt{|\log (T-t)|}\left|\frac{\partial v}{\partial \tau}(\xi, 0)\right| \leq C|f(r)|^{p}$.

$-\tau \in[0,1)$ : We use the equation satisfied by $\frac{\partial v}{\partial \tau}$ and standard tools of localization and local estimates with the semi-group $e^{\tau \Delta}$ to conclude. Indeed, if $z(\xi, \tau)=\left|\frac{\partial v}{\partial \tau}\right|^{2}$, it follows from equation (42) and ii) of lemma 4.1 that $\forall \tau \in[0,1), \forall \xi \in \mathbb{R}$ with $|\xi-\xi(r)| \leq \sqrt{|\log (T-t)|}, \frac{\partial z}{\partial \tau} \leq \Delta z+M z$, where $M=M(p, \delta, N)$.

We can consider $\phi \in \mathcal{C}_{0}^{\infty}(\mathbb{R})$ satisfying $\phi(\xi)=0$ if $|\xi-\xi(r)| \geq$ $\sqrt{|\log (T-t)|}, 0 \leq \phi \leq 1, \phi(\xi)=1$ if $|\xi-\xi(r)| \leq \sqrt{\left|\log \left(T^{\prime}-t\right)\right|} / 2$, and $|\nabla \phi|+|\Delta \phi| \leq C$. 
If $w(\xi, \tau)=e^{-\tau M} \phi(\xi) z(\xi, \tau)$, then $w$ satisfies:

$$
\begin{aligned}
& \frac{\partial w}{\partial \tau} \leq \Delta w+e^{-\tau M}(-z \Delta \phi+2 \nabla z . \nabla \phi) \text { and } \forall \xi \in \mathbb{R},|w(\xi, 0)| \leq C|f(r)|^{2 p} . \\
& \text { If } \tau \in[0,1) \text {, then } \\
& \begin{aligned}
w(\xi(r), \tau) \leq & \left(e^{\tau \Delta} w(0)\right)(\xi(r), \tau) \\
& +\int_{0}^{\tau} \frac{d \sigma}{(4 \pi(\tau-\sigma))^{1 / 2}} \int d x e^{-\frac{|x-\xi(r)|^{2}}{4(\tau-\sigma)}}(z|\Delta \phi|+2|\nabla z||\nabla \phi|)(x, \sigma) \\
\leq & C|f(r)|^{2 p}+\int_{0}^{\tau} \frac{d \sigma}{(4 \pi(\tau-\sigma))^{1 / 2}} \int d x e^{-\frac{|\log (T-t)| / 4}{8(\tau-\sigma)}} e^{-\frac{|x-\xi(r)|^{2}}{8(\tau-\sigma)}} C
\end{aligned}
\end{aligned}
$$

(lemma 4.1 ii) implies by parabolic regularity that for $r \geq r_{2}, t \geq t_{2}(r)$, $(z|\Delta \phi|+2|\nabla z||\nabla \phi|)(x, \sigma) \leq C$, for $\sigma \in[0,1)$ and $|x-\xi(r)| \leq$ $\sqrt{|\log (T-t)|}$.

Therefore, $w(\xi(r), \tau) \leq C|f(r)|^{2 p}+e^{-|\log (T-t)|}$. If $t \geq t_{4}(r)$, then $w(\xi, \tau) \leq C|f(r)|^{2 p}$, which implies $\forall \tau \in[0,1),\left|\frac{\partial v}{\partial \tau}(\xi(r), \tau)\right| \leq C|f(r)|^{p}$.

Taking $r_{1}=\max \left(r_{2}, r_{3}\right)$ and $t_{1}(r)=\max \left(t_{2}(r), t_{3}(r), t_{4}(r)\right)$ concludes the proof.

PART II. - Conclusion of the proof.

For each $r \geq r_{1}$ and each $x \in \mathbb{R} \backslash\{0\}$ small enough, we define $t(r, x) \in[0, T)$ by

$$
|x|=|\xi(r)| \sqrt{T-t}=r \sqrt{(T-t(r, x))|\log (T-t(r, x))|} .
$$

Applying proposition 4.1 to $v(t(r, x))$, we estimate the difference between $u_{*}(x)$ and $u(x, t(r, x))$ and then between $u_{*}(x)$ and $f(r)$. Then, by simple asymptotic calculation, we reach the equivalent (44).

LEMMA 4.2 (A first estimate on the profile $u_{*}(x)$ ). $-\forall r \geq r_{1}, \exists R_{2}(r)>0$ such that $\forall x \in \mathbb{R}$ with $0<|x|<R_{2}$

$$
\left|(T-t(r, x))^{\frac{1+2 \delta}{p-1}} u_{*}(x)-f(r)\right| \leq C|f(r)|^{p},
$$

where $t(r, x)$ is uniquely determined by (45).

Proof. - Using proposition 4.1 and (43), we write for $r \geq r_{1}, t \geq t_{1}(r)$ : $\forall \tau \in[0,1)|v(\xi(r), \tau)-f(r)| \leq|v(\xi(r), \tau)-v(\xi(r), 0)|+\mid v(\xi(r), 0)-$ $\left.f(r)|\leq C| f(r)\right|^{p}+C|\log (T-t)|^{-1 / 2}$. 
Stated in terms of $u$, this gives: $\forall \tau \in[0,1)$

$$
\begin{aligned}
& \left|(T-t)^{\frac{1+i \delta}{p-1}} u(\xi(r) \sqrt{T-t}, t+(T-t) \tau)-f(r)\right| \\
& \quad \leq C|f(r)|^{p}+C|\log (T-t)|^{-1 / 2}
\end{aligned}
$$

From this estimate, we derive $R_{2}(r)>0$ such that $\forall x \in \mathbb{R}$ with $0<|x|<R_{2}$, we have: $\forall \tau \in[0,1) \mid(T-\iota(r, x))^{\frac{1+i \delta}{p-1}} u(x, t(r, x)+(T-$ $t(r, x)) \tau)-\left.f(r)|\leq C| f(r)\right|^{p}$, where $t(r, x)$ is given by (45). If we let $\tau$ go to 1 , we have the conclusion of lemma 4.2.

Now, we conclude the proof of estimate (44). For this purpose, we consider an arbitrary $\varepsilon>0$ and look for $R_{\varepsilon}>0$ such that for $0<|x|<R_{\varepsilon}$,

$$
\left|\left[\frac{|x|^{2}}{-\log |x|}\right]^{\frac{1+i \delta}{p-1}} u_{*}(x)-\left[\frac{8\left(p-\delta^{2}\right)}{(p-1)^{2}}\right]^{\frac{1+i \delta}{p-1}}\right| \leq \varepsilon .
$$

If we consider an arbitrary $r \geq r_{1}$, then by lemma 4.2, we have for $0<|x|<R_{2}$

$$
\begin{aligned}
& \left|\left[\frac{|x|^{2}}{-\log |x|}\right]^{\frac{1+i \delta}{p-1}} u_{*}(x)-\left[\frac{8\left(p-\delta^{2}\right)}{(p-1)^{2}}\right]^{\frac{1+i \delta}{p-1}}\right| \\
& \leq\left|\left[\frac{|x|^{2}}{-\log |x|}\right]^{\frac{1+i \delta}{p-1}}-\left[2 r^{2}(T-t(r, x))\right]^{\frac{1+i \delta}{p-1}}\right| \cdot\left|u_{*}(x)\right| \\
& +\left[2 r^{2}\right]^{\frac{1}{p-1}}\left|(T-t(r, x))^{\frac{1+i \delta}{p-1}} u_{*}(x)-f(r)\right| \\
& +\left|\left[2 r^{2}\right]^{\frac{1+i \delta}{p-1}} f(r)-\left[\frac{8\left(p-\delta^{2}\right)}{(p-1)^{2}}\right]^{\frac{1+i \delta}{p-1}}\right|
\end{aligned}
$$

We fix $r(\varepsilon) \geq r_{1}$ such that $\left|\left[2 r^{2}\right]^{\frac{1+i \delta}{p-1}} f(r)-\left[\frac{8\left(p-\delta^{2}\right)}{(p-1)^{2}}\right]^{\frac{1+i \delta}{p-1}}\right| \leq \varepsilon$ and $|f(r)|^{p-1} \leq \varepsilon$.

From (45), we have

$$
\begin{aligned}
& \frac{|x|^{2}}{-\log |x|} \\
& =2 r^{2}(T-t(r, x)) \frac{\log (T-t(r, x))}{\log (T-t(r, x))+\log |\log (T-t(r, x))|+2 \log r} .
\end{aligned}
$$

Let $R_{\varepsilon}>0$ sufficiently small and smaller than $R_{2}(r(\varepsilon))$ such that for $0<|x|<R_{\varepsilon}$

$$
\left|\left[\frac{|x|^{2}}{-\log |x|}\right]^{\frac{1+i \delta}{p-1}}-\left[2 r^{2}(T-t(r, x))\right]^{\frac{1+i \delta}{p-1}}\right| \leq \varepsilon\left[2 r^{2}(T-t(r, x))\right]^{\frac{1}{p-1}} .
$$


Hence, for $0<|x|<R_{\varepsilon}$, we have from (47):

$$
\begin{aligned}
& \qquad \mid\left[\frac{|x|^{2}}{-\log |x|^{\frac{1+i \delta}{p-1}}} u_{*}(x)-\left[\frac{8\left(p-\delta^{2}\right)}{(p-1)^{2}}\right]^{\frac{1+2 \delta}{p-1}} \mid\right. \\
& \leq \varepsilon\left[2 r^{2}(T-t(r, x))\right]^{\frac{1}{p-1}}\left|u_{*}(x)\right|+C \varepsilon r^{\frac{2}{p-1}}|f(r)|+\varepsilon \\
& \left.\leq C \varepsilon r^{\frac{2}{p-1}}|f(r)|(1+C \varepsilon)+C \varepsilon \text { (use lemma } 4.2 \text { and }|f(r)|^{p-1} \leq \varepsilon\right) \\
& \leq C \varepsilon \text {. This concludes the proof of part iii) of Proposition 1. }
\end{aligned}
$$

\section{GENERALIZATION AND COMMENTS}

As a first application of the techniques in previous sections, we have the following stability result concerning the behavior described in Proposition 1:

THEOREM 3. (Stability with respect to initial data of the profile (4)). Let $\delta \in\left(-\delta_{1}, \delta_{1}\right)$ where $\delta_{1}>0$ and consider $\hat{u}_{0}$ initial data constructed in Proposition I. Let $\vec{u}(t)$ be the solution of equation (I) with initial data $\hat{u}_{0}$, $\hat{T}$ its blow-up time and $\hat{a}$ its blow-up point.

Then there exists a neighborhood $\mathcal{V}$ of $\hat{u}_{0}$ in $H$ with the following properties: For each $u_{0} \in \mathcal{V}, u(t)$ blows-up in finite time $T=T\left(u_{0}\right)$ at one single point $a=a\left(u_{0}\right)$, where $u(t)$ is the solution of equation (1) with initial data $u_{0}$. Moreover, $u(t)$ approaches the profiles (6) and (7) near $(T, a)$ similarly as $\hat{u}(t)$ does near $(\hat{T}, \hat{a})$.

The proof of this theorem relies strongly on the techniques developed in sections 2, 3 and 4 . We give just the key ideas of the proof.

Consider initial data $u_{0}$ in a neighborhood of $\hat{u}_{0}$ and $u(t)$ the corresponding solution of (1). Then, for each $(T, a)$ near $(\hat{T}, \hat{a})$, we introduce as in section 2 a two-parameter group acting on $u(t)$ :

$$
(T, a) \rightarrow(q(T, a, y, s), \theta(T, a, s))
$$

where

$$
\left\{\begin{aligned}
q(T, a, y, s) & =w(T, a, y, s)-\varphi(y, s) \\
\tilde{q}_{2,0}(s) & =0
\end{aligned}\right.
$$

$w(T, a)$ is defined similarly as in (9) by

$$
\begin{aligned}
y & =\frac{x-a}{\sqrt{T-t}} \\
s & =-\log (T-t) \\
w(y, s) & =(T-t)^{\frac{1+i b}{p-1}} u(x, t),
\end{aligned}
$$

and $\varphi$ is given by (16). 
Therefore, our problem reduces to searching a parameter $\left(T\left(u_{0}\right), a\left(u_{0}\right)\right)$ such that

$$
\forall s \geq s_{0}, \quad q(T, a, s) \in V_{A}(s)
$$

for some $s_{0}>0$ and $A>0$ (see definition 3.1). Indeed, $T\left(u_{0}\right)$ and $a\left(u_{0}\right)$ will be shown then to be respectively the blow-up time and point of $u(t)$. Moreover, we derive directly form (48) an estimate analogous to (6) and then, by the techniques of section 4 , an other estimate analogous to (7).

By uniform a priori estimates analogous to proposition 3.2 , we reduce this problem to a finite dimensional one. We solve it using a non-degeneration property of the two-parameter group acting on $\hat{u}(t)$ itself (see [18] for similar argument). Hence, we reach the conclusion of Theorem 3 .

The proof used for equation (1) applies in a more general case: consider the following vector-valued heat equation:

$$
\frac{d u}{d t}=\Delta u+|u|^{p-1} u+G(u), u(x, 0)=u_{0}(x)
$$

where

1) $u(t): x \in \mathbb{R}^{N} \rightarrow \mathbb{R}^{M}, p \in(1,+\infty), p<(N+2) /(N-2)$ if $N \geq 3$,

2) $G: \mathbb{R}^{M} \rightarrow \mathbb{R}^{M}$ is a perturbation of $|u|^{p-1} u$ satisfying: $G(u)=$ $G_{1}\left(|u|^{2}\right) u,|G(u)| \leq C|u|^{r},\left|G\left(\lambda u_{1}\right)-G\left(\lambda u_{2}\right)\right| \leq C \lambda^{r}\left|u_{1}-u_{2}\right|$ for $\left|u_{1}\right|,\left|u_{2}\right| \leq 1, \lambda \geq 1, r \in[1, p), G_{1}: \mathbb{R}^{+} \rightarrow \mathbb{R}^{+}, G$ needs not be a gradient,

3) $u_{0} \in H=W^{1, p+1}\left(\mathbb{R}^{N}, \mathbb{R}^{M}\right) \cap L^{\infty}\left(\mathbb{R}^{N}, \mathbb{R}^{M}\right)$.

Using the same techniques as in the case $M=2$ (equation (1) with $\delta=0$ ), we show the following blow-up result for equation (49):

THEOREM 2 (Existence of a blow-up solution for equation (49)). - There exist initial data $u_{0}$ such that equation (49) has a blow-up solution.

This Theorem is a direct consequence of the following proposition which describes more precisely the behavior of $u(t)$ near blow-up. Indeed, after a time dependent scaling, $u(t)$ approaches a universal profile

$$
\left(p-1+\frac{(p-1)^{2}}{4 p}|z|^{2}\right)^{-\frac{1}{p-1}} \omega
$$

when $t \rightarrow T$, where $\omega \in S^{M-1}$. In fact, we have the more precise result:

PRoposition 2 (Existence of a blow-up solution for equation (49) with the profile (50)). - There exists $T_{0}>0$ such that for each $T \in\left(0, T_{0}\right]$, for each Vol. $15, n^{\circ}$ 5-1998. 
$a \in \mathbb{R}^{N}$, for each $\omega \in S^{M-1}$, there exist initial data $u_{0}$ such that equation (49) has a blow-up solution $u(x, t)$ on $\mathbb{R}^{N} \times[0, T)$ which blows-up in finite time $T$ at only one blow-up point: a. Moreover,

$$
\lim _{t \rightarrow T}(T-t)^{\frac{1}{p-1}} u\left(a+((T-t)|\log (T-t)|)^{\frac{1}{2}} z, t\right)=f(z) \omega
$$

uniformly in $z \in \mathbb{R}^{N}$, with

$$
f(z)=\left(p-1+\frac{(p-1)^{2}}{4 p}|z|^{2}\right)^{-\frac{1}{p-1}} .
$$

Remark Structural stability. - In [18], a particular version of this proposition was shown in the case $M=1$ and $G=0$ (without perturbation): Single point blow-up and a blow-up profile (52). There, this result was shown to be stable with respect to perturbations in initial data. With proposition 2, the blow-up solution constructed in [18] is shown to be structurally stable in a certain class of functions, since this solution behaves in the same way when we take a non zero $G$ and consider a higher dimension $(M \geq 2)$ : we still have single point blow-up with the same scalar profile (52).

\section{A. APPENDIX}

Blow-up result for $\frac{\partial u}{\partial t}=\Delta u+|u|^{p-1} u+i|u|^{q-1} u$ on bounded domain for $q$ small

We consider the complex-valued heat equation (3):

$$
\begin{aligned}
\frac{\partial u}{\partial t} & =\Delta u+|u|^{p-1} u+i|u|^{q-1} u \\
u_{\mid \partial \Omega} & =0
\end{aligned}
$$

where $u(t): \Omega \rightarrow \mathbb{C}, \Omega$ is a bounded domain of $\mathbb{R}^{N}, p \in(1,+\infty)$, $p<(N+2) /(N-2)$ if $N \geq 3$, and $q>1$.

Proposition A.1 (Existence of blow-up solutions for equation (53)). Assume $1<q<(p+1) / 2$. There exists $A(\Omega, p, q)>0$ such that for each $u_{0} \in H_{0}^{1}(\Omega)$ with $\left\|u_{0}\right\|_{L^{2}(\Omega)} \geq A$ and $E\left(u_{0}\right) \leq 0$ where

$$
E\left(u_{0}\right)=\frac{1}{2} \int_{\Omega}\left|\nabla u_{0}\right|^{2} d x-\frac{1}{p+1} \int_{\Omega}\left|u_{0}\right|^{p+1} d x,
$$


equation (53) with initial data $u_{0}$ has a unique solution $u \in$ $\mathcal{C}\left([0, T), H_{0}^{1}(\Omega)\right)$ with $0<T<+\infty$, which blows-up in $H_{0}^{1}(\Omega)$ as $t \rightarrow T$.

Proof. - From classical theory, we know that if $1<q \leq p$ and $u_{0} \in H_{0}^{1}(\Omega)$, then equation (53) with initial data $u_{0}$ has a unique solution defined on $[0, T)$ with $T=T_{u_{0}} \in(0,+\infty]$ and $u \in \mathcal{C}\left([0, T), H_{0}^{1}(\Omega)\right)$. Moreover, if $T<+\infty$, then $u(t)$ blows-up in $H_{0}^{1}(\Omega)$ as $t \rightarrow T$.

Hence, proposition A.1 will be proved if we show that for $1<q<$ $(p+1) / 2,\left\|u_{0}\right\|_{L^{2}(\Omega)}^{2} \geq A$ (to be chosen later) and $E\left(u_{0}\right) \leq 0$, we have $T_{u_{0}}<+\infty$.

We proceed as follows: first we give estimates on $u(t)$ for $t \in[0, T)$, then we use a blow-up result for an integral incquality to conclude.

Lemma A.1 (Estimate for $u(t)$, solution of (53)). - If $z(t)=$ $\left(\int_{\Omega}|u(x, t)|^{p+1} d x\right)^{2 /(p+1)}$, then $\forall t \in[0, T)$,

$$
z(t) \geq c_{1} A^{2}+c_{2} \int_{0}^{t} d \sigma z(\sigma)^{(p+1) / 2}-c_{3} \int_{0}^{t} d \sigma \int_{0}^{\sigma} d s z(s)^{q}
$$

where $c_{1}=c_{1}(\Omega, p)>0, c_{2}=c_{2}(\Omega, p)>0$ and $c_{3}=c_{3}(\Omega, p, q)>0$.

Proof. - For simplicity, we omit $x, \Omega$ and $d x$ in following expressions of the type $\int_{\Omega}|u(x, t)|^{2} d x$.

From (54), $\frac{d}{d t} E(u(t))=\Re\left(-\int \bar{u}_{t}(t) \Delta u(t)-\int|u(t)|^{p-1} u(t) \bar{u}_{t}(t)\right)$.

From (53), $\frac{d}{d t} E(u(t))=\Re\left(-\int \bar{u}_{t}(t) u_{t}(t)+i \int|u(t)|^{q-1} u(t) \bar{u}_{t}(t)\right)$ $\leq-\int\left|u_{t}(t)\right|^{2}+\int|u(t)|^{q}\left|u_{t}(t)\right|$ $\leq-\int\left|u_{t}(t)\right|^{2}+\frac{1}{2}\left(\int\left|u_{t}(t)\right|^{2}+\int|u(t)|^{2 q}\right)$ (Cauchy Schwartz), $\leq-\frac{1}{2} \int\left|u_{t}(t)\right|^{2}+c_{4}(\Omega, p, q)\left(\int|u(t)|^{p+1}\right)^{2 q /(p+1)}$ (Hölder). Integrating this inequality and using $E\left(u_{0}\right) \leq 0$ gives

$$
E(u(t)) \leq c_{4}(\Omega, p, q) \int_{0}^{t} d s\left(\int|u(s)|^{p+1}\right)^{2 q /(p+1)} .
$$

Now, if we multiply equation (53) by $\bar{u}(t)$ and take the real part, we obtain using expression (54)

$$
\frac{d}{d t} \int|u(t)|^{2}=-4 E(u(t))+\frac{p-1}{p+1} \int|u(t)|^{p+1} .
$$

Using (56), $\int|u(0)|^{2} \geq A^{2}$ and $\left(\int|u(t)|^{p+1}\right)^{2 /(p+1)} \geq c_{1}(\Omega, p) \int|u(t)|^{2}$ (Hölder), we have the conclusion by integrating (57). 
Now, the conclusion follows directly from lemma A.1 and the following lemma:

Lemma A.2 (Blow-up result for an integral inequality). - Let $z \in$ $\mathcal{C}\left([0, T), \mathbb{R}^{+}\right)$such that

$$
z(t) \geq B+a \int_{0}^{t} d t^{\prime} z\left(t^{\prime}\right)^{(p+1) / 2}-b \int_{0}^{t} d t^{\prime} \int_{0}^{t^{\prime}} d s z(t)^{q}
$$

where $1<p, 1<q<(p+1) / 2, a>0$ and $b>0$.

There exists $B_{0}>0$ such that if $B \geq B_{0}$, then $T<+\infty$.

Proof. - Let $g(t)=\frac{a}{2} z(t)^{(p+1) / 2}-b \iint_{0}^{t} d s z(s)^{q}$. Let us show that $\forall t \in[0, T), g(t)>0$. We proceed by a priori estimates. For $B>0$, we can define $T^{*}=\sup \left\{T^{\prime} \in[0, T) \mid \forall t \in\left[0, T^{\prime}\right), \int_{0}^{t} d t^{\prime} g\left(t^{\prime}\right) \geq 0\right\}>0$. Then we have $\forall t \in\left[0, T^{*}\right), g(t)>0$.

Indeed, we have $\forall t \in\left[0, T^{*}\right) \int_{0}^{t} d t^{\prime} g\left(t^{\prime}\right) \geq 0$. Therefore, (58) yields $z(t) \geq B+\frac{u}{2} \int_{0}^{t} d s z(s)^{(p+1) / 2}$ which gives $z(t) \geq B$ and $z(t) \geq$ $\frac{a}{2} \int_{0}^{t} d s z(s)^{(p+1) / 2}$. Hence, $g(t)=\frac{a}{2} z(t)^{(p+1) / 2}-b \iint_{0}^{t} d s z(s)^{q}$

$\geq \frac{a}{2} B^{(p-1) / 2} z(t)-b \iint_{0}^{t} d s z(s)^{q}$

$>\frac{a}{2} B^{(p-1) / 2} \frac{a}{2} \int_{0}^{t} d s z(s)^{(p+1) / 2}-b \iint_{0}^{t} d s z(s)^{q}$

$\geq \frac{a^{2}}{4} B^{(p-1) / 2} \int_{0}^{t} d s B^{(p+1) / 2-q} z(s)^{q}-b \iint_{0}^{t} d s z(s)^{q}$

$=\left(\frac{a^{2}}{4} B^{p-q}-b\right) \int_{0}^{t} d s z(s)^{q}$. Now, if $B>\left(4 b a^{-2}\right)^{1 /(p-q)}$, then $\forall t \in\left[0, T^{*}\right)$, $g(t)>0$. This yields $T^{*}=T$ and $\forall t \in[0, T), \int_{0}^{t} d t^{\prime} g\left(t^{\prime}\right) \geq 0$.

Therefore, (58) implies that

$$
\forall t \in[0, T), z(t) \geq B+\frac{a}{2} \int_{0}^{t} d s z(s)^{(p+1) / 2} .
$$

Hence, $T \leq \frac{4 B^{(1-p) / 2}}{a(p-1)}<+\infty$ by classical arguments.

\section{B. APPENDIX}

\section{Proof of lemma 3.3}

Lemma 3.3 consists in a priori estimates on terms appearing in the integral equations satisfied by $\tilde{q}_{1}$ and $\tilde{q}_{2}$ (see (36), (37)). Let us recall them:

$$
\tilde{q}_{1}(s)=K_{1}(s, \sigma) \tilde{q}_{1}(\sigma)+\int_{\sigma}^{s} d \tau K_{1}(s, \tau) V_{1,2}(\tau) \tilde{q}_{2}(\tau)
$$




$$
\begin{aligned}
& +\int_{\sigma}^{s} d \tau K_{1}(s, \tau) \tilde{B}_{1}(q) d \tau+\int_{\sigma}^{s} d \tau K_{1}(s, \tau) \tilde{R}_{1}^{*}(\tau) \\
& +\int_{\sigma}^{s} d \tau K_{1}(s, \tau) \frac{d \theta}{d s}(\tau)\left\{\delta \tilde{\varphi}_{1}(\tau)+\tilde{\varphi}_{2}(\tau)+\delta \tilde{q}_{1}(\tau)+\tilde{q}_{2}(\tau)\right\} \\
\tilde{q}_{2}(s) & =K_{2}(s, \sigma) \tilde{q}_{2}(\sigma)+\int_{\sigma}^{s} d \tau K_{2}(s, \tau) V_{2,1}(\tau) \tilde{q}_{1}(\tau) \\
& +\int_{\sigma}^{s} d \tau K_{2}(s, \tau) \tilde{B}_{2}(q) d \tau+\int_{\sigma}^{s} d \tau K_{2}(s, \tau) \tilde{R}_{2}^{*}(\tau) \\
& -\int_{\sigma}^{s} d \tau K_{2}(s, \tau) \frac{d \theta}{d s}(\tau)\left\{\left(1+\delta^{2}\right) \tilde{\varphi}_{1}(\tau)+\delta \tilde{\varphi}_{2}(\tau)\right)+\left(1+\delta^{2}\right) \tilde{q}_{1}(\tau) \\
& \left.+\delta \tilde{q}_{2}(\tau)\right\}
\end{aligned}
$$

where $K_{1}$ is the fundamental solution of $\mathcal{L}+V_{1,1}, K_{2}$ is the fundamental solution of $\mathcal{L}-1+V_{2,2}, \mathcal{L}$ is given by (30), $B(q)=(1+i \delta) \tilde{B}_{1}+i \tilde{B}_{2}$, $R^{*}(y, s)=(1+i \delta) \tilde{R}_{1}^{*}+i \tilde{R}_{2}^{*}, B$ and $R^{*}$ are given by $(20)$.

From these expressions, we obviously see that the main step in doing a priori estimates is the understanding of the behavior of the kernels $K_{1}$ and $K_{2}$. By definition, $K_{1}$ and $K_{2}$ can be considered as perturbations of $e^{\theta \mathcal{L}}$ and $e^{\theta(\mathcal{L}-1)}$ respectively. Hence, we give the proof in two steps:

- in Step 1, we give estimates on the integral operators $K_{1}$ and $K_{2}$, nonlinear term $B(q)$ and corrective term $R^{*}$ appearing in equations (36) and (37).

- in Step 2, we use these estimates to prove lemma 3.3.

StEP 1. - Estimates on linear, nonlinear and corrective terms of (36) and (37).

In order to estimate $K_{1}$ and $K_{2}$, we follow the perturbation method used in [18] (and before in Bricmont and Kupiainen [4]). Since $K_{1}$ and $K_{2}$ correspond respectively to the operators $\mathcal{L}+V_{1,1}$ and $\mathcal{L}-1+V_{2,2}$, we estimate first the potentials $V_{i, j}$ so we are able to adapt the cited method which compares $K_{1}$ and $K_{2}$ to $e^{\theta \mathcal{L}}$ and $e^{\theta(\mathcal{L}-1)}$ respectively. Then, we show that $B(q)$ can be considered in some sense as a quadratic term, and $R^{*}$ is in fact small as $s \rightarrow+\infty$.

Lemma B.1 (Estimates on potentials $V_{i, j},|\delta| \leq 1 / 2$ ). $-\forall s \geq 1$,

a) $V_{1,1}(y, s) \leq C s^{-1},\left|\frac{d^{n} V_{1,1}}{d y^{n}}\right| \leq C s^{-n / 2}, n=0,1,2$, 
$\left|V_{1,1}(y, s)\right| \leq C s^{-1}\left(1+|y|^{2}\right), V_{1,1}(y, s)=-\frac{1}{4 s} h_{2}(y)+\tilde{V}_{1,1}(y, s)$ with

$\left|\tilde{V}_{1,1}(y, s)\right| \leq C s^{-2}\left(1+|y|^{4}\right), \forall \varepsilon>0, \exists C_{\varepsilon}^{\prime}>0, \exists s_{\varepsilon}$ such that

$$
\sup _{s \geq s_{\varepsilon}, \frac{|y|}{\sqrt{s}} \geq C_{\varepsilon}}\left|V_{1,1}(y, s)-\left(-\frac{p-\delta^{2}}{p-1}\right)\right| \leq \varepsilon
$$

with $-\frac{p-\delta^{2}}{p-1} \leq-1-1 /(2 p)$.

b) $V_{2,2}(y, s) \leq C s^{-1},\left|\frac{d^{n} V_{2,2}}{d y^{n}}\right| \leq C s^{-n / 2}, n=0,1,2$,

$\left|V_{2,2}(y, s)\right| \leq C s^{-1}\left(1+|y|^{2}\right), V_{2,2}(y, s)=s^{-1} Q_{\delta}(y)+\tilde{V}_{2_{2}}(y, s)$ with $Q_{\delta}$ a polynomial of degree 2 with bounded coefficients and $\left|\tilde{V}_{2,2}(y, s)\right| \leq$ $C s^{-2}\left(1+|y|^{4}\right)$

$\forall \varepsilon>0, \exists C_{\varepsilon}>0, \exists s_{\varepsilon}$ such that

$$
\sup _{s \geq s_{\varepsilon}, \frac{|y|}{\sqrt{s}} \geq C_{\varepsilon}}\left|-1+V_{2,2}(y, s)-\left(-1-\frac{1 \mid \delta^{2}}{p-1}\right)\right| \leq \varepsilon
$$

with $-1-\frac{1+\delta^{2}}{p-1}<-1-1 / p$.

c) For $V=V_{1,2}$ or $V_{2,1}$, we have $|V(y, s)| \leq C|\delta|$, and $|V(y, s)| \leq$ $C|\delta| s^{-1}\left(1+|y|^{2}\right)$.

Proof. - The expressions of $V_{i, j}$ are given in lemma 3.2 .

a) $\quad V_{1,1}(y, s) \leq\left(1-\delta^{2}\right)\left(|\varphi(0, s)|^{p-1}-\frac{1}{p-1}\right)+(p-$ 1) $|\varphi(0, s)|^{p-3}\left(|\varphi(0, s)|^{2}-0\right)-1 \sim C(\delta) s^{-1} \leq C s^{-1}$.

We introduce $W_{1,1}(z, s)=V_{1,1}(y, s)$ with $z=y / \sqrt{s}$. In order to prove the next estimate, it is enough to prove that $\left|\frac{d^{n} W_{2,2}}{d y^{n}}\right| \leq C, n=0,1,2$. Since $V_{1,1}$ is a sum of products of terms $|\varphi|^{p-1}$ and $\varphi_{j} /|\varphi|, j=1,2$, our problem reduces to proving that these terms have bounded first and second derivatives with respect to $z$, which follows easily (see (16), the key estimates are $\frac{\partial f_{\delta}}{\partial z}=\frac{-2 b z}{(p-1)\left(p-1+b z^{2}\right)} f_{\delta}$ and $\left|f_{\delta}\right| \leq|\varphi|$ with $b=\frac{(p-1)^{2}}{4\left(p-\delta^{2}\right)}$ ).

We introduce $\tilde{W}_{1,1}(Z, s)=V_{1,1}(y, s)$ with $Z=|y|^{2} / s$. We can Taylor expand $\tilde{W}_{1,1}$ near $Z=0$ to have $\tilde{W}_{1,1}(Z, s)=\tilde{W}_{1,1}(0, s)+$ $Z \frac{\partial \tilde{W}_{1,1}}{\partial Z}(0, s)+O\left(Z^{2}\right)$ with $\tilde{W}_{1,1}(0, s)=1 /(2 s)+O\left(s^{-2}\right)$ and $\frac{\partial \tilde{W}_{1,1}}{\partial Z}(0, s)=$ $-1 / 4$ । $O\left(s^{-1}\right)$. Returning to $V_{1,1}$, this yields the next two estimates.

The last estimate is obvious from the expressions of $V_{1,1}$ and $\varphi$.

b) For the first term, we make a change of variables by setting $Y=$ $\kappa^{-1} f_{\delta}(y / \sqrt{s})+1 /\left(2\left(p-\delta^{2}\right) s\right) \in\left(1 /\left(2\left(p-\delta^{2}\right) s\right), 1 /\left(2\left(p-\delta^{2}\right) s\right)+1\right]$ and $\hat{V}_{2,2}(Y, s)=V_{2,2}(y, s)$. Then, it is easy to see that $\hat{V}_{2,2}(., s)$ is increasing. Therefore, $\hat{V}_{2,2}(Y, s) \leq \hat{V}_{2,2}\left(1 /\left(2\left(p-\delta^{2}\right) s\right)+1, s\right) \sim C(\delta) s^{-1} \leq C s^{-1}$. For next estimates, do exactly as for $V_{1,1}$. 
c) Same proofs, one has to be careful with the parameter $\delta$.

LEMMA B.2 ( Estimates on $K_{1},|\delta|<1 / 2$ ).

a) $\forall s \geq \tau>1$ with $s \leq 2 \tau, \forall y, x \in \mathbb{R},\left|K_{1}(s, \tau, y, x)\right| \leq C e^{(s-\tau) \mathcal{L}}(y, x)$, with

$e^{\theta \mathcal{L}}(y, x)=\frac{e^{\theta}}{\sqrt{4 \pi\left(1-e^{-\theta}\right)}} \exp \left[-\frac{\left(y e^{-\theta / 2}-x\right)^{2}}{4\left(1-e^{-\theta}\right)}\right]$,

$\left\|K_{1}(s, \tau)(1-\chi(\tau))\right\|_{L^{\infty}} \leq C e^{-(s-\tau) /(2 p)}$.

b) For each $A^{\prime}>0, A^{\prime \prime}>0, A^{\prime \prime \prime}>0, \rho^{*}>0$, there exists $s_{9}\left(A^{\prime}, A^{\prime \prime}, A^{\prime \prime \prime}, \rho^{*}\right)$ with the following property:

$\forall s_{0} \geq s_{9}$, assume that for $\sigma \geq s_{0}$,

$$
\begin{aligned}
\left|q_{m}(\sigma)\right| & \leq A^{\prime} \sigma^{-2}, m=0,1,\left|q_{2}(\sigma)\right| \leq A^{\prime \prime}(\log \sigma) \sigma^{-2} \\
\left|q_{-}(y, \sigma)\right| & \leq A^{\prime \prime \prime}\left(1+|y|^{3}\right) \sigma^{-2},\left\|q_{e}(\sigma)\right\|_{L^{\infty}} \leq A^{\prime \prime} \sigma^{-\frac{1}{2}}
\end{aligned}
$$

then, $\forall s \in\left[\sigma, \sigma+\rho^{*}\right]$

$$
\begin{aligned}
\left|\alpha_{2}(s)\right| & \leq A^{\prime \prime} \frac{\log \sigma}{s^{2}}+(s-\sigma) C A^{\prime} s^{-3} \\
\left|\alpha_{-}(y, s)\right| & \leq C\left(e^{-\frac{1}{2}(s-\sigma)} A^{\prime \prime \prime}+e^{-(s-\sigma)^{2}} A^{\prime \prime}\right)\left(1+|y|^{3}\right) s^{-2} \\
\left\|\alpha_{e}(s)\right\|_{L^{\infty}} & \leq C\left(A^{\prime \prime} e^{-\frac{(s-\sigma)}{p}}+A^{\prime \prime \prime} e^{(s-\sigma)}\right) s^{-\frac{1}{2}}
\end{aligned}
$$

where, as in decomposition (22),

$K_{1}(s, \sigma) q(\sigma)=\alpha(y, s)=\sum_{m=0}^{2} \alpha_{m}(s) h_{m}(y)+\alpha_{-}(y, s)+\alpha_{e}(y, s)$

c) For each $A^{\prime}>0, A^{\prime \prime}>0, A^{\prime \prime \prime}>0, \rho^{*}>0$, there exists $s_{10}\left(A^{\prime}, A^{\prime \prime}, A^{\prime \prime \prime}, \rho^{*}\right)$ with the following property:

$\forall s_{0} \geq s_{10}$, assume that for $\sigma \geq s_{0}$

$$
\begin{aligned}
\left|q_{m}(\sigma)\right| & \leq A^{\prime} \sigma^{-2}, m=0,1,\left|q_{2}(\sigma)\right| \leq A^{\prime \prime} \sigma^{-3} \\
\left|q_{-}(y, \sigma)\right| & \leq A^{\prime \prime \prime}\left(1+|y|^{3}\right) \sigma^{-3},\left\|q_{e}(\sigma)\right\|_{L^{\infty}} \leq A^{\prime} \sigma^{-2}
\end{aligned}
$$

then, $\forall s \in\left[\sigma, \sigma+\rho^{*}\right]$

$$
\begin{aligned}
\left|\alpha_{2}(s)\right| & \leq A^{\prime \prime} s^{-3}+(s-\sigma) C A^{\prime} s^{-3} \\
\left|\alpha_{-}(y, s)\right| & \leq C A^{\prime \prime \prime}\left(1+|y|^{3}\right) s^{-3}
\end{aligned}
$$

where $K_{1}(s, \sigma) q(\sigma)$ is expanded in (59).

Proof. - In [4] (proof of lemma 1), the authors prove the estimate for an integral operator $K$ corresponding to $\mathcal{L}+V$ (see (30) for $\mathcal{L}$ ), where $V$ Vol. 15, n ${ }^{\circ}$ 5-1998. 
is a particular function. However, their result is in fact true for a larger class of operators satisfying estimates of the type $a$ ) in lemma B.1. Hence, lemma B.2 follows.

Lemma B.3 (Estimates on $K_{2},|\delta|<1 / 2$ ).

a) $\forall s \geq \tau>1$ with $s \leq 2 \tau, \forall y, x \in \mathbb{R}$,

$\left|K_{2}(s, \tau, y, x)\right| \leq C e^{-(s-\tau)} e^{(s-\tau) \mathcal{L}}(y, x)$, with

$e^{\theta \mathcal{L}}(y, x)=\frac{e^{\theta}}{\sqrt{4 \pi\left(1-e^{-\theta}\right)}} \exp \left[-\frac{\left(y e^{-\theta / 2}-x\right)^{2}}{4\left(1-e^{-\theta}\right)}\right]$,

$\left\|K_{2}(s, \tau)(1-\chi(\tau))\right\|_{L^{\infty}} \leq C e^{-(s-1) / p}$.

b) For each $A^{\prime}>0, A^{\prime \prime}>0, \rho^{*}>0$, there exists

$s_{11}\left(A^{\prime}, A^{\prime \prime}, \rho^{*}\right)$ with the following property:

$\forall s_{0} \geq s_{11}$, assume that for $\sigma \geq s_{0}$,

$$
\begin{aligned}
\left|q_{0}(\sigma)\right| & \leq A^{\prime} \sigma^{-2}, m=0,1,\left|q_{\perp}(y, \sigma)\right| \leq A^{\prime}\left(1+|y|^{3}\right) \sigma^{-2}, \\
\left\|q_{e}(\sigma)\right\|_{L^{\infty}} & \leq A^{\prime \prime} \sigma^{-\frac{1}{2}}
\end{aligned}
$$

then, $\forall s \in\left[\sigma, \sigma+\rho^{*}\right]$

$$
\begin{aligned}
\left|\alpha_{\perp}(y, s)\right| & \leq C\left(e^{-\frac{1}{2}(s-\sigma)} A^{\prime}+e^{-(s-\sigma)^{2}} A^{\prime \prime}\right)\left(1+|y|^{3}\right) s^{-2}, \\
\left\|\alpha_{e}(s)\right\|_{L^{\infty}} & \leq C\left(A^{\prime \prime} e^{-\frac{(s-\sigma)}{p}}+A^{\prime}\right) s^{-\frac{1}{2}},
\end{aligned}
$$

where, as in decomposition (23),

$$
K_{2}(s, \sigma) q(\sigma)=\alpha(y, s)=\alpha_{0}(s) h_{0}(y)+\alpha_{\perp}(y, s)+\alpha_{e}(y, s) .
$$

Proof. - Again, we can adapt the proof of lemma 1 in [4] with $\mathcal{L}$ replaced by $\mathcal{L}-1$ and $V$ replaced by $V_{2,2}$, without difficulties. Indeed, one checks easily that $V_{2,2}$ satisfies all useful estimates: $b$ ) of lemma B.1.

LEMMA B.4 (Estimates on $B(q(\tau))$ for $q(\tau)$ in $V_{A}(\tau)|\delta| \leq 1 / 2$ ) $\forall A>0, \exists s_{12}(A)>0$ such that $\forall \tau \geq s_{12}(A), q(\tau) \in V_{A}(\tau)$ implies $|\chi(y, \tau) B(q(y, \tau))|=\left|(1+i \delta) \chi \tilde{B}_{1}+i \chi \tilde{B}_{2}\right| \leq C|q|^{2}$, $|B(q)|=\left|(1+i \delta) \tilde{B}_{1}+i \tilde{B}_{2}\right| \leq C|q|^{\bar{p}}$ with $\bar{p}=\min (p, 2)$.

Proof. - Start with (20) and do the same as in the proof of lemma 3.15 in $[18]$.

Lemma B.5 (Estimates on $\left.R^{*}(y, s),|\delta| \leq 1 / 2\right)$. $\forall s \geq 1$, if $R^{*}$ is expanded as in (24), then:

$\left|\tilde{R}_{1,0}^{*}(s)\right| \leq C s^{-2}, \tilde{R}_{1,1}^{*}(s)=0,\left|\tilde{R}_{1,2}^{*}(s)\right| \leq C s^{-3}$, 
$\left|\tilde{R}_{1,-}^{*}(y, s)\right| \leq C s^{-2}\left(1+|y|^{3}\right),\left\|\tilde{R}_{1, e}^{*}(s)\right\|_{L^{\infty}} \leq C s^{-1}$, and $\left|\tilde{R}_{2,0}^{*}(s)\right| \leq C s^{-2},\left|\tilde{R}_{2, \perp}^{*}(y, s)\right| \leq C s^{-2}\left(1+|y|^{3}\right),\left\|\tilde{R}_{2, e}^{*}(s)\right\|_{L^{\infty}} \leq C s^{-1}$.

Proof. $-\tilde{R}_{1,1}^{*}(s)=0$ since $R^{*}$ is even. All the other estimates follow from the three following estimates: $\left|\chi(y, s) R^{*}(y, s)\right| \leq C s^{-2}\left(1+|y|^{2}\right)$, $\left|R^{*}(y, s)\right| \leq C s^{-1}$ and $\left|\tilde{R}_{1,2}^{*}(s)\right| \leq C s^{-3}$.

Proof of $\left|\chi(y, s) R^{*}(y, s)\right| \leq C s^{-2}\left(1+|y|^{2}\right)$

From (20), we have

$$
\begin{aligned}
R^{*}(y, s) & \\
= & \frac{\partial \varphi}{\partial s}+\Delta \varphi-\frac{1}{2} y \cdot \nabla \varphi-(1+i \delta) \frac{\varphi}{p-1}+(1+i \delta)|\varphi|^{p-1} \varphi \\
= & -(1+i \delta) \kappa^{-i \delta}\left(f_{\delta}+\frac{\kappa}{2\left(p-\delta^{2}\right) s}\right)^{i \delta}\left(-\frac{\kappa}{2\left(p-\delta^{2}\right) s^{2}}+\frac{(p-1) y^{2}}{4\left(p-\delta^{2}\right) s^{2}} f_{\delta}^{p}\right) \\
+ & (1+i \delta) \kappa^{-i \delta}\left(f_{\delta}+\frac{\kappa}{2\left(p-\delta^{2}\right) s}\right)^{i \delta} \frac{(p-1) y^{2}}{4\left(p-\delta^{2}\right) s} f_{\delta}^{p} \\
+ & (1+i \delta) i \delta \kappa^{-i \delta}\left(f_{\delta}+\frac{\kappa}{2\left(p-\delta^{2}\right) s}\right)^{i \delta-1}\left(\frac{(p-1) y}{2\left(p-\delta^{2}\right) s} f_{\delta}^{p}\right)^{2} \\
+ & (1+i \delta) \kappa^{-i \delta}\left(f_{\delta}+\frac{\kappa}{2\left(p-\delta^{2}\right) s}\right)^{i \delta} \\
& \times\left(-\frac{(p-1)}{2\left(p-\delta^{2}\right) s} f_{\delta}^{p}+\frac{p(p-1)^{2} y^{2}}{4\left(p-\delta^{2}\right)^{2} s^{2}} f_{\delta}^{2 p-1}\right) \\
+ & (1+i \delta) \kappa^{-i \delta}\left(\left(f_{\delta}+\frac{\kappa}{2\left(p-\delta^{2}\right) s}\right)^{p+i \delta}\right. \\
& \left.-\frac{1}{p-1}\left(f_{\delta}+\frac{\kappa}{2\left(p-\delta^{2}\right) s}\right)^{1+i \delta}\right) .
\end{aligned}
$$

Some of these terms are easily seen to be bounded by $C s^{-2}\left(1+|y|^{2}\right)$, whereas others need some calculation: we divide the others by $(1+i \delta)\left(f_{\delta}+\right.$ $\left.\frac{\kappa}{2\left(p-\delta^{2}\right) s}\right)^{i \delta} \kappa^{-i \delta}$ and obtain $Q(y, s)=\frac{(p-1) y^{2}}{4\left(p-\delta^{2}\right) s} f_{\delta}^{p}-\frac{(p-1)}{2\left(p-\delta^{2}\right) s} f_{\delta}^{p}-\frac{1}{p-1}\left(f_{\delta}+\right.$ $\left.\frac{\kappa}{2\left(p-\delta^{2}\right) s}\right)+\left(f_{\delta}+\frac{\kappa}{2\left(p-\delta^{2}\right) s}\right)^{p}$. It remains to prove that $|\chi(y, s) Q(y, s)| \leq$ $C s^{-2}\left(1+|y|^{2}\right)$. We write $Q(y, s)=\left(f_{\delta}+\frac{\kappa}{2\left(p-\delta^{2}\right) s}\right)^{p}-f_{\delta}^{p}-\frac{(p-1)}{2\left(p-\delta^{2}\right) s} f_{\delta}^{p}-$ $\frac{\kappa}{2\left(p-\delta^{2}\right)(p-1) s}$. Setting $z=\frac{|y|^{2}}{s} \geq 0$ and $\hat{Q}(z, s)=Q(y, s)$, we have $|\hat{Q}(0, s)| \leq C s^{-2}$ and $\left|\frac{\partial \hat{Q}}{\partial z}(z, s)\right|=p \mid \frac{\partial f_{\delta}}{\partial z}\left\{\left(f_{\delta}+\frac{\kappa}{2\left(p-\delta^{2}\right) s}\right)^{p-1}-f_{\delta}^{p-1}-\right.$ $\left.\frac{(p-1)}{2\left(p-\delta^{2}\right) s} f_{\delta}^{p-1}\right\} \mid \leq C s^{-1}$ if $z \leq 2 K_{0}$, (Taylor expansion). Therefore, if $z \leq 2 K_{0},|\hat{Q}(z, s)| \leq C s^{-2}+O\left(|z| s^{-1}\right)$. Returning to $Q$, this gives the result. 
Proof of $\left|R^{*}(y, s)\right| \leq C s^{-1}$. - Thinking of $R^{*}$ as a function of $|y|^{2} s^{-1}$ and $s$ (see (60)), this estimate is obvious for all terms except $(1+i \delta) \kappa^{-i \delta}\left(f_{\delta}+\frac{\kappa}{2\left(p-\delta^{2}\right) s}\right)^{i \delta}\left(\frac{(p-1)|y|^{2}}{4\left(p-\delta^{2}\right) s} f_{\delta}^{p}+\left(f_{\delta}+\frac{\kappa}{2\left(p-\delta^{2}\right) s}\right)^{p}-\frac{1}{p-1} f_{\delta}\right)=$ $(1+i \delta) \kappa^{-i \delta}\left(f_{\delta}+\frac{\kappa}{2\left(p-\delta^{2}\right) s}\right)^{i \delta}\left(\left(f_{\delta}+\frac{\kappa}{2\left(p-\delta^{2}\right) s}\right)^{p}-f_{\delta}^{p}\right)$. We conclude using a Taylor expansion.

Proff of $\left|\tilde{R}_{1,2}^{*}(s)\right| \leq C s^{-3}-$ From (60), we have

$$
\tilde{R}_{1}^{*}(y, s)=-\frac{\partial \varphi_{1}}{\partial s}+\Delta \varphi_{1}-\frac{1}{2} y \cdot \nabla \varphi_{1}+\left(|\varphi|^{p-1}-\frac{1}{p-1}\right)\left(\varphi_{1}-\delta \varphi_{2}\right) .
$$

Starting from $\tilde{R}_{1,2}^{*}(s)=\int d \mu(y) \chi(y, s) \tilde{R}_{1}^{*}(y, s) \frac{h_{2}(y)}{8}$, one carries out easy but long asymptotic calculation to get the result.

STEP 2. - Conclusion of the proof of lemma 3.3.

We now prove lemma 3.3 .

I i) Case $\sigma \geq s_{0}$ : Apply $b$ ) of lemma B.2 with $A^{\prime}=A, A^{\prime \prime}=A^{2}$ and $A^{\prime \prime \prime}=A$.

Case $\sigma=s_{0}$ : From (25), $\tilde{q}_{1}\left(y, s_{0}\right)=f_{0}\left(\frac{y}{\sqrt{s_{0}}}\right)^{p}\left(d_{0}+d_{1} y / \sqrt{s_{0}}\right)-\Re\left(\left(\frac{\kappa}{2\left(p-\delta^{2}\right) s_{0}}\right)^{1+i \delta}\right)$. Since $\left(d_{0}, d_{1}\right)$ is chosen so that $\left(\tilde{q}_{1,0}\left(s_{0}\right), \tilde{q}_{1,1}\left(s_{0}\right)\right) \in \hat{V}_{A}\left(s_{0}\right)$, we have from lemma 3.5 in [18], $\left|\tilde{q}_{1, m}\left(s_{0}\right)\right| \leq A s_{0}^{-2}, m=0,1,\left|\tilde{q}_{1,2}\left(s_{0}\right)\right| \leq\left(\log s_{0}\right) s_{0}^{-2}$, $\left|\tilde{q}_{1,-}\left(y, s_{0}\right)\right| \leq C s_{0}^{-2}\left(1+|y|^{3}\right)$ and $\left\|\tilde{q}_{1, e}\left(s_{0}\right)\right\|_{L^{\infty}} \leq s_{0}^{-1 / 2}$. We apply b) of lemma B.2 with $A^{\prime}=A, A^{\prime \prime}=C, A^{\prime \prime \prime}=1$ to conclude

I ii): We have from lemma B. $\left|V_{1,2}(y, s)\right| \leq C|\delta| s^{-1}\left(1+|y|^{2}\right)$.

Since $q(\tau) \in V_{A}(\tau),\left|V_{1,2}(y, \tau) \tilde{q}_{2}(y, \tau)\right| \leq C A|\delta| \tau^{-3}\left(1+|y|^{4}\right)$.

Hence, $\left|\iota_{1,2}(s)\right|=\left|C \int d \mu h_{2}(y) \int_{\sigma}^{s} d \tau K_{1}(s, \tau) V_{1,2}(\tau) \tilde{q}_{2}(\tau)\right|$

$\leq C \int d \mu\left(1+|y|^{2}\right) \int_{\sigma}^{s} d \tau e^{(s-\tau) \mathcal{L}} C A|\delta| \tau^{-3}\left(1+|x|^{4}\right)$

$\leq C A|\delta| \sigma^{-3} \int d \mu\left(1+|y|^{6}\right)(s-\sigma) e^{s-\sigma}$

$\leq C A|\delta| s^{-3}(s-\sigma) e^{s-\sigma}$, if $\sigma \geq s_{0} \geq \rho^{*}$.

If we set $Q(y, \tau)=V_{1,2}(y, \tau) \tilde{q}_{2}(y, \tau)$, we have by lemma B.1 vij $\left|V_{1,2}(y, \tau)\right| \leq C|\delta|$ and then $\left|Q_{m}(\tau)\right| \leq C|\delta| A \tau^{-2}, m=0,1,2$, $\left|Q_{-}(y, \tau)\right| \leq C|\delta| A\left(1+|y|^{3}\right) \tau^{-2},\left|Q_{e}(y, \tau)\right| \leq C|\delta| A^{2} \tau^{-1 / 2}$. Applying lemma B.2 and integrating between $\sigma$ and $s$ yields good estimates for $\iota_{1,-}$ and $\iota_{1, e}$.

I iii): Using lemma B.2 and a) of lemma B.2, we do the same as for the nonlinear term in Proof of lemma 3.12 in [18].

I iv): From lemma B.5, we have $\left|\tilde{R}_{1,0}^{*}(\tau)\right| \leq C \tau^{-2}, \tilde{R}_{1,1}^{*}(\tau)=0$, $\left|\tilde{R}_{1,2}^{*}(\tau)\right| \leq C \tau^{-3},\left|\tilde{R}_{1,-}^{*}(y, \tau)\right| \leq C \tau^{-2}\left(1+|y|^{3}\right),\left|\tilde{R}_{1, e}^{*}(y, \tau)\right| \leq C \tau^{-1}$. 
Applying lemma B.5 $b$ ) and integrating between $\sigma$ and $s$ gives the results for $\gamma_{1,2}$ and $\gamma_{1,-}$.

For $\gamma_{1, e}$,we use the following estimate: $\left|R^{*}(y, \tau)\right| \leq C \tau^{-1}$, and compute: $\left|\gamma_{1, e}\right|=\left|\int_{\sigma}^{s} d \tau K_{1}(s, \tau) R_{1}^{*}(\tau)\right|$ $\leq \int_{\sigma}^{s} d \tau e^{(s-\sigma) \mathcal{L}} C \tau^{-1}$ (use lemma B.2 a)) $\leq C \sigma^{-1}(s-\sigma) e^{s-\sigma} \leq C s^{-3 / 4}(s-\sigma)$ if $s_{0} \geq s_{5}\left(\rho^{*}\right)$.

I v): We set $Q(y, \tau)=\frac{d \theta}{d s}(\tau)\left\{\delta \tilde{q}_{1}+\tilde{q}_{2}+\delta \tilde{\varphi}_{1}+\tilde{\varphi}_{2}\right\}$. By lemma 3.1, we have $\left|\frac{d \theta}{d s}(\tau)\right| \leq C \tau^{-2}$. Using $q(\tau) \in V_{A}(\tau), \varphi$ bounded and a simple calculation, we have:

$\left|Q_{m}(\tau)\right| \leq C A \tau^{-2}, m=0,1,\left|Q_{2}(\tau)\right| \leq C|\delta| \tau^{-3},\left|Q_{-}(y, \tau)\right| \leq$ $C A\left(1+|y|^{3}\right) \tau^{-3},\left|Q_{e}(y, \tau)\right| \leq C_{\tau^{-2}}$.

Using lemma B.2 c), we obtain estimates for $\lambda_{1,2}$ and $\lambda_{1,-}$. For $\lambda_{1, e}$, use $|Q(y, \tau)| \leq C \tau^{-2}$ and do as for $\gamma_{1, e}$.

II i): For $\sigma \geq s_{0}$, use lemma B.3.

For $\sigma=s_{0}$, we have from (25)

$\tilde{q}_{2}\left(y, s_{0}\right)=\frac{\alpha}{s_{0}}\left(\delta \cos \left[\delta \log \left(\frac{\alpha}{s_{0}}\right)\right]-\sin \left[\delta \log \left(\frac{\alpha}{s_{0}}\right)\right]\right)\left(1-\beta\left(s_{0}\right) f_{0}\left(\frac{y}{\sqrt{s_{0}}}\right)\right)$ where $\alpha$ and $\beta\left(s_{0}\right)$ are given by (26). It follows easily that $\tilde{q}_{2,0}\left(s_{0}\right)=0$, $\left|\tilde{q}_{2, \perp}\left(y, s_{0}\right)\right| \leq C s_{0}^{-2}\left(1+|y|^{3}\right)$ and $\left|\tilde{q}_{2, e}\left(y, s_{0}\right)\right| \leq C s_{0}^{-1} \leq s_{0}^{1 / 2}$. Apply b) of lemma B.3 to conclude.

II ii): we have by lemma B.1 $\left|V_{2,1}(y, \tau)\right| \leq C|\delta|$ and $\left|V_{2,1}(y, \tau)\right| \leq$ $C|\delta| \tau^{-1}\left(1+|y|^{2}\right)$. If $Q(y, \tau)=V_{2,1}(y, \tau) \tilde{q}_{1}(y, \tau)$, then $\left|Q_{0}(\tau)\right| \leq C|\delta| A^{2} s^{-3} \log s,\left|Q_{\perp}(y, \tau)\right| \leq C|\delta| A s^{-2}$ and $\left|Q_{e}(y, \tau)\right| \leq C|\delta| A^{2} s^{-1 / 2}$.

Using lemma B.3 b) yields the conclusion.

II iii): Using lemmas B.4 and lemma B.3 a), we do the same as for I iii). II iv): Same estimates as Iiv).

II v): By lemma 3.1, we have $\left|\frac{d \theta}{d s}(\tau)\right| \leq C \tau^{-2}$. Using lemma B.3 $a$ ) and integrating over $[\sigma, s]$ yields the conclusion.

\section{REFERENCES}

[1] J. BALL, Remarks on blow-up and nonexistence theorems for nonlinear evolution equations, Quart. J. Math. Oxford, Vol. 28, 1977, pp. 473-486.

[2] M. BERGER and R. KOHN, A rescaling algorithm for the numerical calculation of blowing-up solutions, Comm. Pure Appl. Math., Vol. 41, 1988, pp. 841-863.

[3] J. BRICMONT and A. KuPIAINEN, Renormalization group and nonlinear PDEs, Quantum and non-commutative analysis, past present and future perspectives, Kluwer (Boston), 1993.

[4] J. BRicmont and A. KuPIAINEN, Universality in blow-up for nonlinear heat equations, Nonlinearity, 7, 1994, pp. 539-575. 
[5] S. FilipPas and R. KOHN, Refined asymptotics for the blowup of $u_{t}-\Delta u=u^{\prime}$, Comm. Pure Appl. Math., Vol. 45, 1992, pp. 821-869

[6] S. FilipPas and F. MERLE, Modulation theory for the blowup of vector-valued nonlinear heat equations, J. Diff. Equations, Vol. 116, 1995, pp. 119-148.

[7] V. A. Galaktionov, S. P. Kurdyumov and A. A. Samarski, On approximate self-similar solutions for some class of quasilinear heat equations with sources, Math. USSR-Sb, Vol. 52, 1985, pp. 155-180.

[8] V. A. Galaktionov and J. L. VazQUez, Regional blow-up in a semilinear heat equation with convergence to a Hamilton-Jacobi equation, SIAM J. Math. Anal., Vol. 24, 1993, pp. 1254-1276.

[9] Y. GIGA and R. KOHN, Asymptotically self-similar blowup of semilinear heat equations, Comm. Pure Appl. Math., Vol. 38, 1985, pp. 297-319.

[10] Y. Giga and R. KoHn, Characterizing blowup using similarity variables, Indiana Univ. Math. J., Vol. 36, 1987, pp. 1-40.

[11] Y. GiGA and R. KoHn, Nondegeneracy of blow-up for semilinear heat equations, Comm. Pure Appl. Math., Vol. 42, 1989, pp. 845-884.

[12] R. S. Hamilton, The formation of singularities in the Ricci flow, Surveys in differential geometry, Vol. II, Internat. Press, Cambridge, 1995, pp. 7-136.

[13] M. A. HerRero and J. J. L. VELAZQUeZ, Blow-up behavior of one-dimensional semilinear parabolic equations, Ann. Inst. Henri Poin-caré, Vol. 10, 1993, pp. 131-189.

[14] M. A. Herrero and J. J. L. VelazQuez, Flat blow-up in one-dimensional semilinear heat equations, Differential and Integral eqns., Vol. 5, 1992, pp. 973-997.

[15] C. D. Levermoke and M. OlJver, The complex Gincburg-Landau equation as a ruodel problem, Dynamical systems and probabilistic methods in partial differential equations (Berkeley, 1994), Lectures in Appl. Math., Vol. 31, Amer. Math. Soc., Providence, RI, 1996, pp. 141-190.

[16] H. LEvINE, Some nonexistence and instability theorems for solutions of formally parabolic equations of the form $P u_{i}=-A u+F(u)$, Arch. Rat. Mech. Anal., Vol. 51, 1973, pp. 371-386.

[17] F. MERLE, Solution of a nontinear heat equation with arbitrary given blow-up points, Comm. Pure Appl. Math., Vol. 45, 1992, pp. 263-300.

[18] F. MERLE and H. ZAAG, Stability of blow-up profile for equation of the type $u_{t}=$ $\Delta u+|u|^{p-1} u$, preprint.

[19] J. J. L. VelazQuez, Classification of singularities for blowing up solutions in higher dimensions, Trans. Amer. Math. Soc., Vol. 338, 1993, pp. 441-464.

[20] F. WEISSLER, Single-point blowup for a semilinear initial value problem, J. Diff. Equations, Vol. 55, 1984, pp. 204-224.

(Manuscript received June 21, 1996.) 